\title{
Brain development in the yellow fever mosquito Aedes aegypti: a comparative immunocytochemical analysis using cross-reacting antibodies from Drosophila melanogaster
}

\author{
Keshava Mysore • Susanne Flister • Pie Müller • \\ Veronica Rodrigues $\cdot$ Heinrich Reichert
}

Received: 1 April 2011 /Accepted: 14 September 2011 /Published online: 30 September 2011

(C) Springer-Verlag 2011

\begin{abstract}
Considerable effort has been directed towards understanding the organization and function of peripheral and central nervous system of disease vector mosquitoes such as Aedes aegypti. To date, all of these investigations have been carried out on adults but none of the studies addressed the development of the nervous system during the larval and pupal stages in mosquitoes. Here, we first screen a set of 30 antibodies, which have been used to study brain development in Drosophila, and identify 13 of them cross-reacting and labeling epitopes in the developing brain of Aedes. We then use the identified antibodies in immunolabeling studies to characterize general neuroanatomical features of the developing brain and compare them with the well-studied
\end{abstract}

Communicated by V. Hartenstein

Electronic supplementary material The online version of this article (doi:10.1007/s00427-011-0376-2) contains supplementary material, which is available to authorized users.

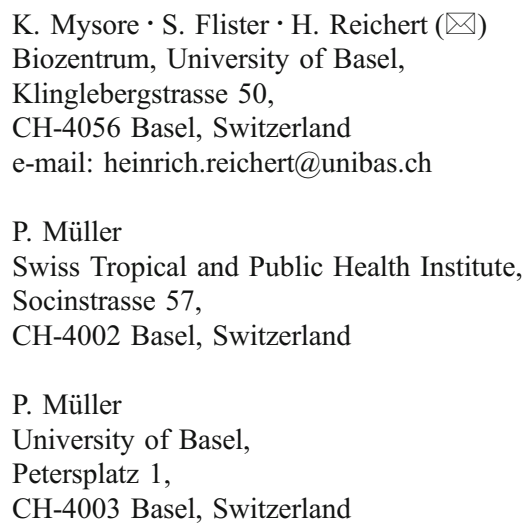

\section{Rodrigues}

National Centre for Biological Sciences, Tata Institute for Fundamental Research, GKVK Campus,

Bellary Road,

Bangalore 560065, India model system, Drosophila melanogaster, in larval, pupal, and adult stages. Furthermore, we use immunolabeling to document the development of specific components of the Aedes brain, namely the optic lobes, the subesophageal neuropil, and serotonergic system of the subesophageal neuropil in more detail. Our study reveals prominent differences in the developing brain in the larval stage as compared to the pupal (and adult) stage of Aedes. The results also uncover interesting similarities and marked differences in brain development of Aedes as compared to Drosophila. Taken together, this investigation forms the basis for future cellular and molecular investigations of brain development in this important disease vector.

Keywords Aedes aegypti . Fruitfly · Brain development . Optic lobes $\cdot$ Subesophageal ganglion

\section{Introduction}

Mosquitoes are vectors for major parasitic diseases and viral infections such as malaria, dengue, yellow fever, and encephalitis by feeding on blood from infected individuals and transferring the disease agents to naïve hosts; hence, control of the mosquito vectors is an important goal for global health (Feachem et al. 2010). Host-seeking behavior in mosquitoes is based on chemosensation involving the peripheral olfactory and gustatory sense organs as well as the central olfactory and gustatory circuitry of the brain. Since basic knowledge about the organization, function, and development of olfactory and gustatory systems in mosquitoes may improve the design of existing and allows to design novel disease control strategies (e.g., repellents, traps), there has been considerable effort directed towards understanding the organization and function of the periph- 
eral and central nervous system in mosquitoes (Anton et al. 2003; Ignell et al. 2005; Ignell and Hansson 2005; Kwon et al. 2006; Ghaninia et al. 2007a, b; Lu et al. 2007; Siju et al. 2008; Wang et al. 2010; Carey et al. 2010). These structural and functional studies of the nervous system have been complemented by molecular analyses of the mosquito olfactory and gustatory receptor molecules and, in more general terms, by the sequencing of the genomes of several mosquito species (Fox et al. 2001; Hill et al. 2002; Melo et al. 2004; Bohbot et al. 2007; Lu et al. 2007; Arensburger et al. 2010). While a wealth of studies has investigated the adult's olfactory system, studies elucidating the development of the sensory receptors or the central nervous circuitry involved in mosquito chemosensation during the larval stages are critically absent. As a consequence, virtually nothing is known about the development of the chemosensory system in mosquitoes.

In other insects such as the fruit fly, Drosophila melanogaster, the hawk moth, Manduca sexta, as well as in social insects such as ants and bees, molecular tools (e.g., antibodies) have been very useful for understanding the development of peripheral and central chemosensory systems (Oland and Tolbert 1996; Rodrigues and Hummel 2008; Groh and Rössler 2008; Mysore et al. 2010). Notably, in the genetic model Drosophila, a wealth of monoclonal and polyclonal antibodies generated against numerous molecular antigens involved in nervous system development and function are currently available and have been used extensively to understand the various aspects of peripheral and central nervous system development, including olfactory system development (reviewed by Rodrigues and Hummel 2008). In neuroanatomical respects, a major finding in Drosophila brain development is the fact that an identified set of neuroblasts generates all of the neurons in the central brain in a lineage-specific manner. Most of the lineally related neurons that derive from a given neuroblast have comparable morphological features and their axons come together to form coherent fascicles that project together. Since a given lineage of neurons has a common projection trajectory, lineages therefore form "units of projection" that underlie connectivity in the brain (Larsen et al. 2009; Pereanu et al. 2011). An important open question is whether this lineage-based organizational principle also holds for other Dipteran insects.

Although fruitflies and mosquitoes are Dipteran insects, the two insect groups diverged about 250 million years ago and, thus, manifest considerable differences reflecting adaptation associated with different ecological niches and life strategies (Yeates and Wiegmann 1999; Gaunt and Miles 2002). Nevertheless, it has been suggested that more than half of the genes in their genomes (Drosophila versus Anopheles) are putative orthologs with an average sequence identity of approximately 56\% (Zdobnov et al. 2002). In view of this molecular genetic similarity, it seems likely that many of the antibody tools that were generated and used in the study of the development of the nervous system in Drosophila might cross-react with the corresponding epitopes and, thus, be useful molecular labels for investigating mosquito brains. Indeed, in two cases, antibodies generated/used in Drosophila have been shown to crossreact in adult mosquito nervous systems, and they have been utilized to understand the general neuroanatomy of the adult brain as well as specific types of neurons in the adult chemosensory system of mosquitoes (Ignell et al. 2005; Ghaninia et al. 2007a, b; Siju et al. 2008). Taken together, these findings suggest that antibodies generated in Drosophila might be very helpful tools for understanding the development of the mosquito brain.

To obtain a suite of antibody markers that can be used to understand the various processes involved in the development of the mosquito brain in general (and the olfactory system in particular), we analyzed a set of 30 antibodies, which have been used to study brain development in Drosophila, for cross-reactivity in the developing brains of mosquito Aedes aegypti. Here, we identify 13 of these antibodies that cross-react and label epitopes in the developing mosquito brain. We then characterize the general neuroanatomical features of the mosquito brain revealed by each of these cross-reacting antibodies in immunolabeling studies in larval, pupal and the adult mosquitoes and compare these features with those of fruit fly brains labeled with same antibodies. Moreover, using these antibodies, we analyze the development of specific components of the brain (optic lobes, subesophageal neuropil, and serotonergic system of subesophageal neuropil) in Aedes. Our analysis reveals prominent differences in the neuroanatomy of the brain in the larval stage as compared to the pupal and the adult stages. Our findings also document remarkable similarities and major differences in brain development of Aedes as compared to Drosophila. Taken together, these findings form a basis for subsequent cellular and molecular studies of brain development in the important disease vector Aedes.

\section{Material and methods}

\section{Mosquito rearing}

Eggs of A. aegypti Rockefeller strain were collected from a stock cage were reared at $27^{\circ} \mathrm{C}$ with about $70 \%$ humidity. The hatchlings were collected and reared separately at same conditions and were fed with TetraMin ${ }^{\circledR}$ Baby (Tetra $\mathrm{GmbH}$, Melle, Germany) fish food. For the dissections and antibody cross-reactivity assays, mosquitoes were 
collected as fourth instar larvae, pupae ( $24 \mathrm{~h}$ after puparium formation (APF)) pupae and 1-day-old adults.

\section{Fly strains}

The Oregon R wild type strain was used for all the Drosophila experiments. Flies were reared in $25^{\circ} \mathrm{C}$ in $12 \mathrm{~h} \mathrm{LD}$ cycle. For the larval experiments, the wandering third-stage larvae were collected, for the pupal experiments the pupae were collected at $0 \mathrm{H} \mathrm{APF}$ and were staged to $45-50 \mathrm{~h} \mathrm{APF}$ at $25^{\circ} \mathrm{C}$. The adults that were 1-day-old after eclosion were used for the adult brain analyses; for consistency, only female adults were used for immunohistochemistry.

\section{Immunohistochemistry}

The protocol described by Ignell et al. (2005) and Ghaninia et al. (2007a) was modified for screening of cross-reactivity of various antibodies on mosquito brains. Briefly, individuals of various stages were immobilized on ice for about $30 \mathrm{~min}$, then the brains were dissected in chilled $1 \times$ phosphate-buffered saline (PBS) and fixed overnight in $2 \%$ PFA diluted in $1 \%$ PTx at $4{ }^{\circ} \mathrm{C}$. Then the samples were washed in $1 \%$ PTx $\left(15^{\prime} \times 4\right)$ and were pre-incubated at room temperature in $10 \%$ NGS solution prepared in 1\% PTx for $1 \mathrm{~h}$. As a next step, the antibodies were diluted in the pre-incubation solution to the desired concentrations and incubated for $48 \mathrm{~h}$ at $4^{\circ} \mathrm{C}$. The samples were then washed in $1 \%$ PTx $\left(15^{\prime} \times 4\right)$ and incubated in Alexa Fluor 488/568 (Molecular Probes, Invitrogen Corporation, CA, USA) secondary antibodies; the preparations were incubated overnight at $4^{\circ} \mathrm{C}$ and washed in $1 \%$ PTx $\left(15^{\prime} \times 4\right)$ and mounted in Vectashield (Vector Laboratories, CA, USA) mounting medium. For immunohistochemical localization of serotonin in the central nervous system (CNS) of all the stages (larvae, pupae, and adults), the protocol described by Siju et al. (2008) was followed. For staining the Drosophila brain, the protocol mentioned earlier (Bello et al. 2007) was used. Briefly, brains of larvae, pupae and adult were dissected in chilled $1 \times$ PBS, fixed in $2 \%$ paraformaldehyde in PBL $(75 \mathrm{mM}$ lysine $\mathrm{HCl}$ in sodium phosphate buffer, $\mathrm{pH}$ 7.4) for $1 \mathrm{~h}$ at room temperature. Later, they were washed in $0.5 \% \mathrm{PTx}$ and were incubated in the primary antibody overnight and in the secondary antibody for $4 \mathrm{~h}$ at room temperature. In case of adults, the brains were incubated in primary and secondary antibodies for 2 days at $4{ }^{\circ} \mathrm{C}$ for best results. The antibodies were diluted in the pre-incubation solution with the dilution mentioned below. In both the cases, a nuclear counterstain was done using TOTO-3 iodide (Molecular Probes, Invitrogen Corporation, CA, USA), to mark the boundary of the individual brains along with the antibody of interest.
The following antibodies were screened for cross-reactivity against the mosquito CNS: mouse anti-SYNORF 1 (1:10, DSHB); mouse BP 102 (1:5, DSHB); mouse 7E8A10 (1:10, DSHB); mouse 8D12 (1:10, DSHB); mouse nc82 (1:100, Pielage Lab, FMI, Basel, Switzerland); mouse 22 C10 (1:50, DSHB); mouse BP 104 (1:1, DSHB); rat DN-EX\#8 (1:10, DSHB); mouse N2 7A1 (1:100, DSHB); mouse 7 G10 (1:10, DSHB); mouse E7 (1:50, DSHB); mouse 24B10 (1:50, DSHB); mouse 9F8A9 (1:10, DSHB); mouse anti-tubulin, acetylated (1:100, Sigma); mouse anti- $\gamma$ Tubulin $(1: 1,000$, Sigma); mouse anti-tubulin, tyrosine (1:500, Sigma); rabbit anti-5HT (1:1000, Sigma) rabbit anti-horseradish peroxidase (HRP; 1:500, AbCam); rabbit anti- $\alpha$ tubulin (1:500, AbCam); rabbit anti- $\beta$ III Tubulin (1:200, AbCam); rabbit antiengrailed (1:50, Santa Cruz); goat anti-engrailed (1:50, Santa Cruz); rabbit anti- $\alpha$ tubulin, acetylated (1:500, CST); rabbit anti-anti-phospho histone 3 (PH3; 1:200, Upstate); mouse antiglutamine synthetase (1:200,) rat anti-Repo (1:20, Rodrigues Lab, NCBS-TIFR, Bangalore, India); rabbit anti-Repo (1:500, Technau Lab, Univ. of Mainz, Mainz, Germany); guinea pig anti-Deadpan (1:500, Skeath Lab, Washington University at St. Louis, USA) and rat anti-Deadpan (undiluted, Lee lab, University of Michigan, USA). A detailed description of the antibodies that were used and those cross-reacted against the mosquito brain are provided as Supplementary Table 1 and Table 1 . As a control for the specificity of the antibodies, the secondary antibody was applied in the absence of the primary antibody and in no case any staining was observed (data not shown). Of the aforementioned antibodies, only the crossreacting antibodies were used to do a comparative analysis of the fruit fly brain (Table 1).

\section{Confocal imaging and image processing}

Stained whole mount preparations were visualized through a Leica SP5 confocal microscope (Leica Microsystems, Germany). Stacks of 80-100 images were collected at step sizes of $1.0 \mu \mathrm{m}$ (10× objectives for whole brain) with 1.3 $1.5 \times$ digital zoom at a resolution of $1,024 \times 1,024$ pixels. Preparations labeled with Alexa 488 were excited with an argon laser at $488 \mathrm{~nm}$ wave length and fluorescence detected through a $505 \mathrm{~nm}$ long-pass filter. The nuclear marking dye TOTO-3 was excited using a $\mathrm{HeNe}$ laser at $633 \mathrm{~nm}$ and detected using a $650 \mathrm{~nm}$ filter. The images were exported as TIFF images from the LAS AF offline software and analyzed using Fiji (http://pacific.mpi-cbg.de/wiki/index.php/Fiji) and Photoshop CS4 (Adobe Systems, CA, USA). In all the cases (Figs. 1, 2, 3, 4, 5, and 6), a single hemisphere counterstained with TOTO- 3 is used to represent the staining pattern and the major neuropils are labeled wherever identified. In addition, six supplementary figures are provided containing the high-resolution images in gray scale from all cross-reacting antibodies in the Aedes brain. 
Table 1 Antibodies that cross-reacted against the developing brains of Aedes aegypti

\begin{tabular}{|c|c|c|c|c|c|c|c|}
\hline \multirow[t]{2}{*}{ Sl. no } & \multirow[t]{2}{*}{ Name of the $\mathrm{Ab}$} & \multirow[t]{2}{*}{ Antigen } & \multirow[t]{2}{*}{ Host } & \multicolumn{3}{|l|}{ Result } & \multirow[t]{2}{*}{ Concentration } \\
\hline & & & & Larvae & Pupae & Adult & \\
\hline 1 & Anti SYNORF 1 & Synapsin (Drosophila) & Mouse & + & + & + & 1 in 10 \\
\hline 2 & nc82 & Bruchpilot (Drosophila) & Mouse & + & + & + & 1 in 100 \\
\hline 3 & $22 \mathrm{C} 10$ & Neurons (Drosophila) & Mouse & + & + & + & 1 in 50 \\
\hline 4 & DN-EX \# 8 & DN-cadherin (Drosophila) & Rat & - & + & + & 1 in 10 \\
\hline 5 & E7 & $\beta$-galactosidase (Escherichia coli) & Mouse & + & + & + & 1 in 50 \\
\hline 6 & T9028 & Tubulin, tyrosine (C-terminal of $\alpha$-tubulin) & Mouse & + & + & + & 1 in 500 \\
\hline 7 & S5545 & Serotonin creatinine sulfate complex & Rabbit & + & + & + & 1 in 1000 \\
\hline 8 & ab2115 & Horseradish peroxidase & Rabbit & + & + & + & 1 in 500 \\
\hline 9 & ab15246 & $\alpha$-Tubulin (synthetic peptide-aa426 to 450 ) & Rabbit & + & + & + & 1 in 500 \\
\hline 10 & $5335 \mathrm{~S}$ & Acetylated- $\alpha$-tubulin (synthetic peptide) & Rabbit & + & + & + & 1 in 100 \\
\hline 11 & $05-789$ & Phospho histone H3 (synthetic peptide) & Rabbit & + & - & - & 1 in 200 \\
\hline 12 & 610518 & Glutamine synthetase (sheep) & Mouse & + & + & + & 1 in 200 \\
\hline 13 & - & Repo (Drosophila) & Rabbit & + & + & + & 1 in 500 \\
\hline
\end{tabular}

Of the 30 antibodies from fruit fly and other sources that were screened for cross-reactivity against the developing brain of the mosquito $A$. aegypti, 14 cross-reacted positively (indicated by "+" sign) marking various compartments of the mosquito brain. Certain antibodies showed stage specific expression ("-" sign indicates the stages where they were not expressed/did not cross react with that particular stage)

\section{Results}

Identification of cross-reacting antibodies in Aedes

Of the total of 30 antibodies screened, 13 antibodies cross-react with the developing CNS of mosquito the A. aegypti (Table 1; Supplementary Table 1). Among these, seven antibodies have been generated against neuronal or glial epitopes; the remaining six have been generated against various other defined epitopes. The known immunolabeling properties of these 13 antibodies in the insect CNS are as follows.

The monoclonal mAb3C11 (anti-SYNORF-1) antibody labels synaptic boutons in Drosophila and hence strongly stains most of the synaptic neuropil in the fruit fly CNS (Klagges et al. 1996). Apart from Drosophila, it has been shown to cross-react in various invertebrate nervous systems (Ignell et al. 2005; Ghaninia et al. 2007a; Groh and Rössler 2008; Mysore et al. 2010). The monoclonal mAb nc82 antibody labels the entire neuropil in Drosophila and has been extensively used to characterize anatomical features of the Drosophila CNS (Rein et al. 2002). The monoclonal mAb $22 \mathrm{C} 10$ antibody labels the sensory neurons in Drosophila including those of the visual system (Zipursky et al. 1984). The monoclonal anti-Repo antibody (raised against the glial-specific REPO homeoprotein) labels most glial cells in the Drosophila CNS (Halter et al. 1995). The polyclonal anti-glutamine synthetase (GS) antibody is known to label subsets of astrocyte-like glial cells, in several invertebrate and vertebrate species (MartinezHernandez et al. 1977; van der Hel et al. 2005; Ward et al. 2004; Harzsch and Hansson 2008). The anti-Cadherin (DN-
EX\#8) antibody, raised against a Drosophila DN-cadherin epitope, is strongly expressed in axons of the fruit fly CNS (Iwai et al. 1997). The polyclonal anti-serotonin (anti-5HT) antibody labels this neurotransmitter in numerous species; it has previously been shown to label various compartments of the central and peripheral chemosensory system in the adults of A. aegypti (Dacks et al. 2006) and Anopheles gambiae (Siju et al. 2008). Four antibodies generated against different tubulin epitopes, namely the anti-tubulin (acetylated) antibody, the anti- $\beta$-tubulin antibody, the anti- $\alpha$-tubulin antibody, and anti-tubulin (tryrosinylated) antibody have been used to label the nervous systems of different species of arthropods (Chitnis and Kuwada 1990; Eriksson and Budd 2000; Mayer et al. 2010). The polyclonal anti-HRP antibody, raised against the enzyme HRP, recognizes several epitopes on neuronal processes and has been used as a "pan-neuronal" label in Drosophila, grasshopper and other insects (Snow et al. 1987). Finally, the PH3 antibody, raised against phosphorylated threonine 11 of histone 3 from human, marks cells in mitosis in many species (Preuss et al. 2003).

In view of their differential labeling properties in the Drosophila and other invertebrate nervous system, we reasoned that these 13 cross-reacting antibodies should allow immunohistological visualization of diverse morphological aspects in the Aedes brain, and that they should also reveal differences in the organization of the brain at different life cycle stages in this insect. In the following, we take advantage of the specific immunolabeling properties of these antibodies to characterize the major features of the Aedes brain in the larva (fourth larval instar stage (L4)), in the pupa (24 $\mathrm{h} \mathrm{APF),} \mathrm{and} \mathrm{in} \mathrm{the} \mathrm{adult.} \mathrm{Further,} \mathrm{we} \mathrm{compare} \mathrm{these}$ 


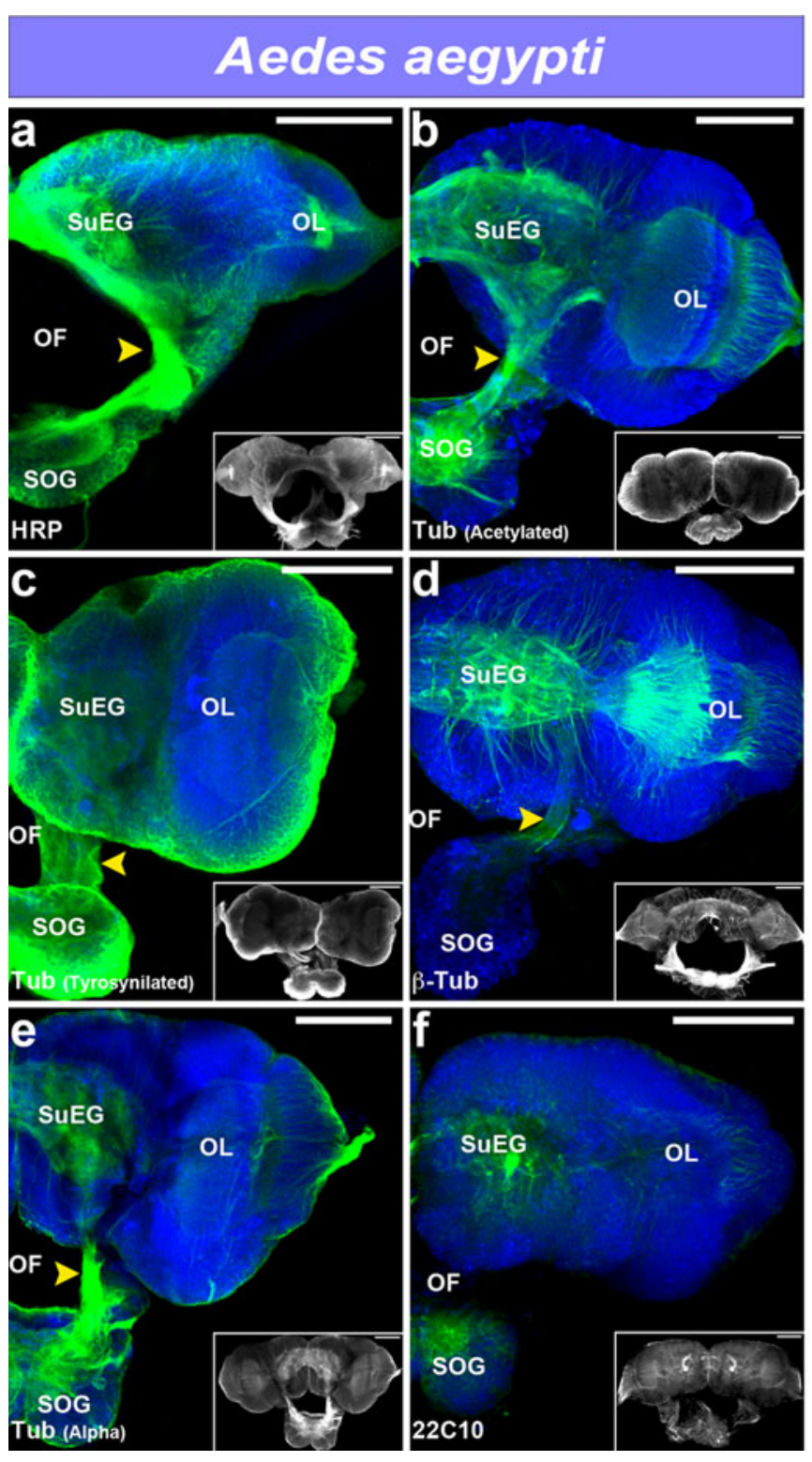

Fig. 1 Cross-reacting antibodies against the larval brain (stage L4) of $A$. aegypti label similar compartmental structures as observed in the fruit fly larval brain. Information on the overall organization of the larval brain is provided by the anti-HRP antibody $\left(\mathbf{a}, \mathbf{a}^{\prime}\right)$ and by the 4 antitubulin antibodies (b-e and $\left.\mathbf{b}^{\prime}-\mathbf{e}^{\prime}\right)$. Anti-22 C10 immunostaining reveal comparable aspects of the larval brain both in cortical and neuropil

results with the immunolabeling patterns of the same 13 antibodies on the developing larval stage (third-stage larva), pupal stage (45-50 h APF), and the adult of the fruit fly Drosophila.

Organization of the developing brain in the Aedes larva stage

Information on the overall organization of the developing Aedes brain in the larval stage (L4) is provided by the "pan neuronal" anti-HRP antibody and by the four anti-tubulin

\section{Drosophila melanogaster}
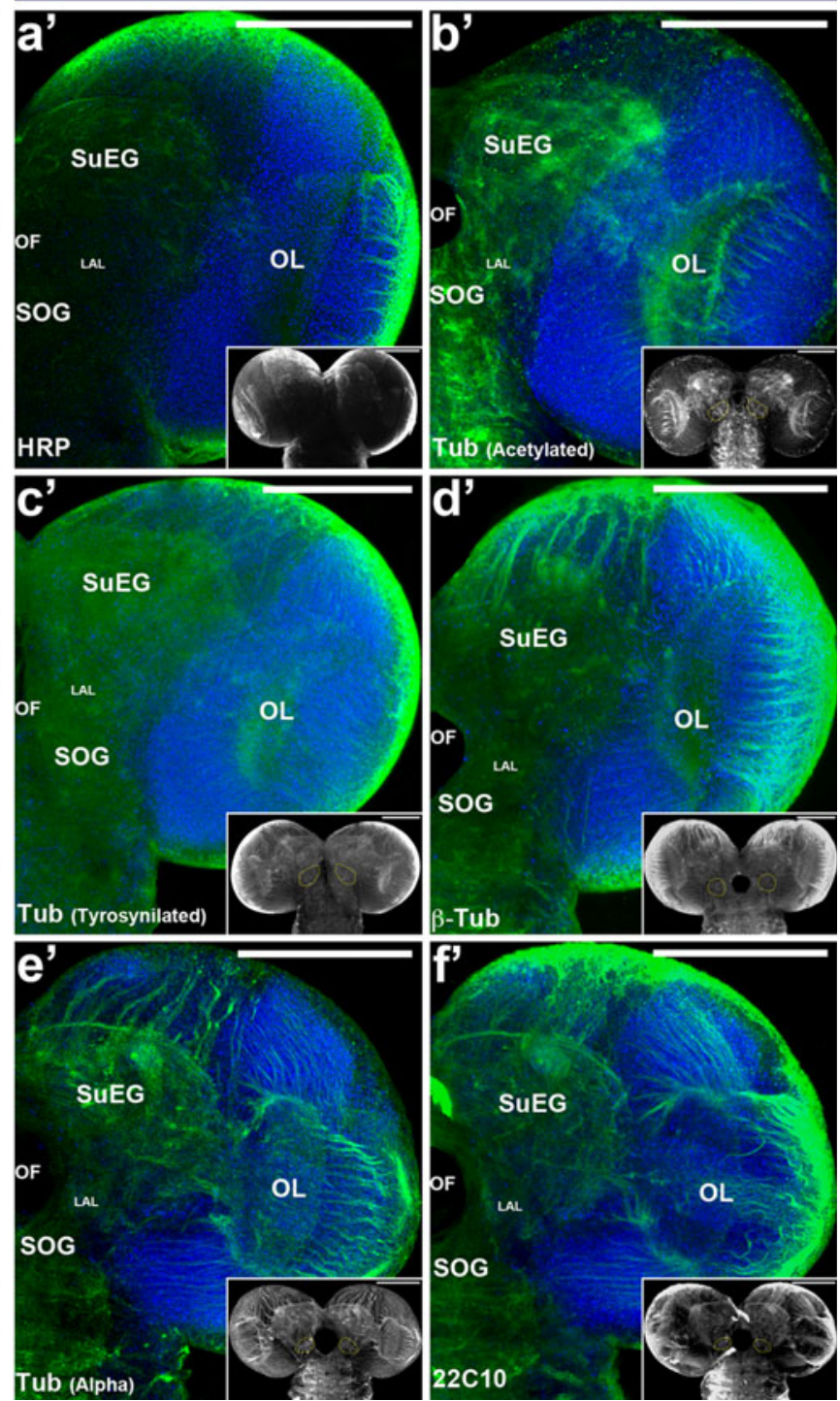

regions (f, f'). $L A L$ larval antennal lobe, $O F$ esophageous foramen, $O L$ optic lobe, $S O G$ subesophageal ganglion, $S u E G$ supraesophageal ganglion. Yellow arrowhead indicates the connector between SuEG and SOG in Aedes brain. Scale bar $100 \mu \mathrm{m}$. The label on the left bottom of each image corresponds to the antibody represented

antibodies. These antibodies reveal comparable cellular and neuroanatomical features when applied to the fruit fly larval brain (third instar). In Aedes, anti-HRP immunoreactivity reveals the entire brain composed of a large supraesophageal ganglion that is connected by descending connectives (Fig. 1a) to a smaller subesophageal ganglion and flanked laterally by optic lobes that receive input from the larval compound eyes. This antibody when applied to fruit fly brain reveals similar aspects; it labels the nerve bundles of the optic lobes very intensely and it also marks the nerve bundles in the cortical regions (Fig. 1a'). Noteworthy in the 


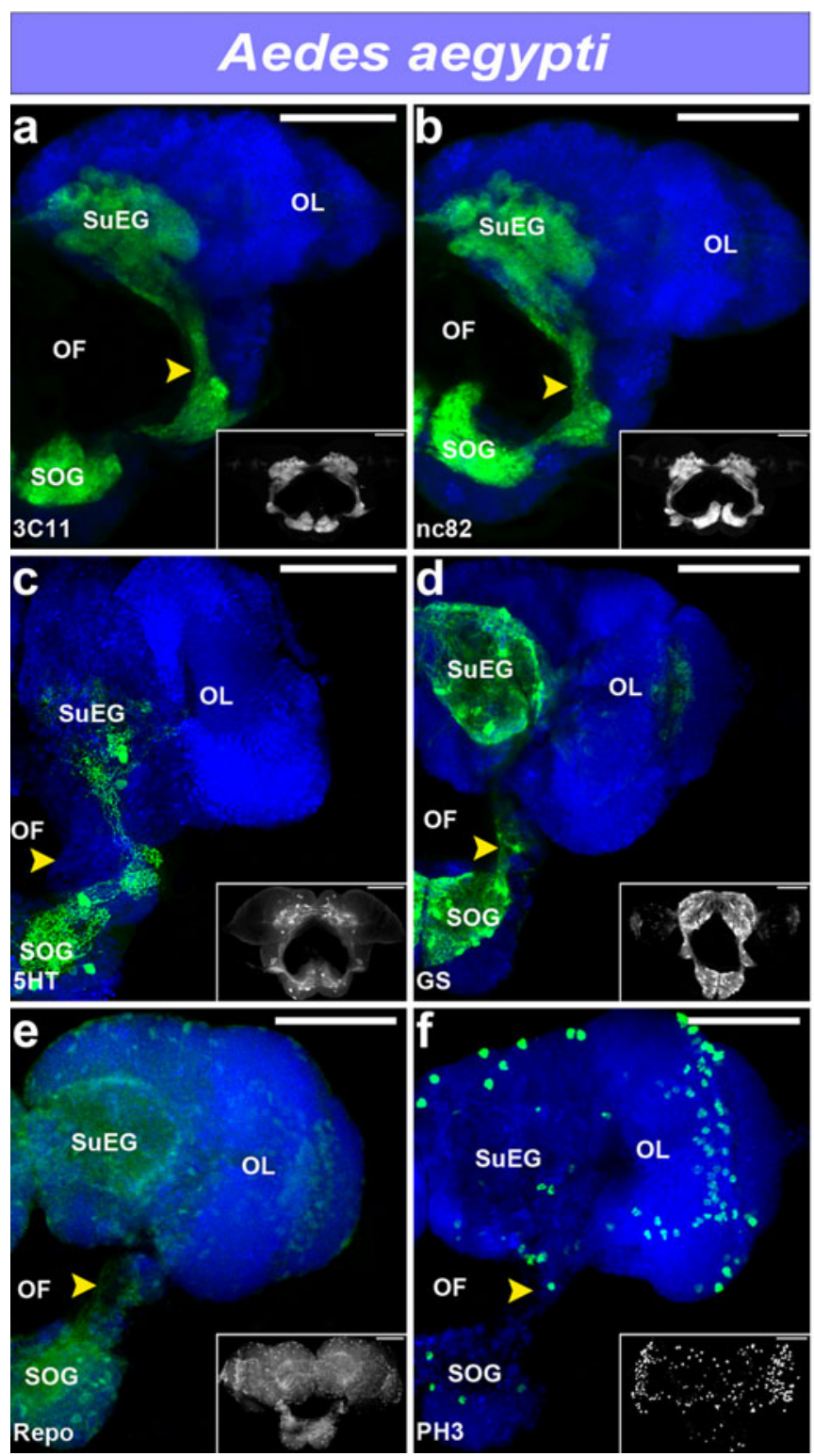

Fig. 2 Cross-reacting antibodies against the larval brain (stage L4) of $A$. aegypti label similar compartmental structures as observed in the fruit fly larval brain. Anti-3 C11 (a, $\left.\mathbf{a}^{\prime}\right)$ and anti-nc82 (b, b') immunostaining reveals a complex compartment-like organization in the neuropil of the supraesophageal and subesophageal ganglia. anti-5HT immunostaining (c, $\left.\mathbf{c}^{\prime}\right)$ labels the 5HT immunoreactive cell bodies as well as their projections in the neuropil, commissures and connectives of the supraesophageal and subesophageal ganglia. Anti-GS and anti-Repo

Aedes mosquito is that the supraesophageal ganglion and the subesophageal ganglia are not fused and that there is a large central space between them that corresponds to the esophagus foramen. In contrast in the fruit fly larva, the entire brain is apparent as a fused structure consisting of a two-lobed larval brain without any obvious anatomically apparent subdivision into a supraesophageal and a subesophageal ganglion (Figs. $1 a^{\prime}-f^{\prime}$ and $2 a^{\prime}-f^{\prime}$ ). Anti-tubulin (acetylated) immunolabeling highlights the neuropil regions

\section{Drosophila melanogaster}

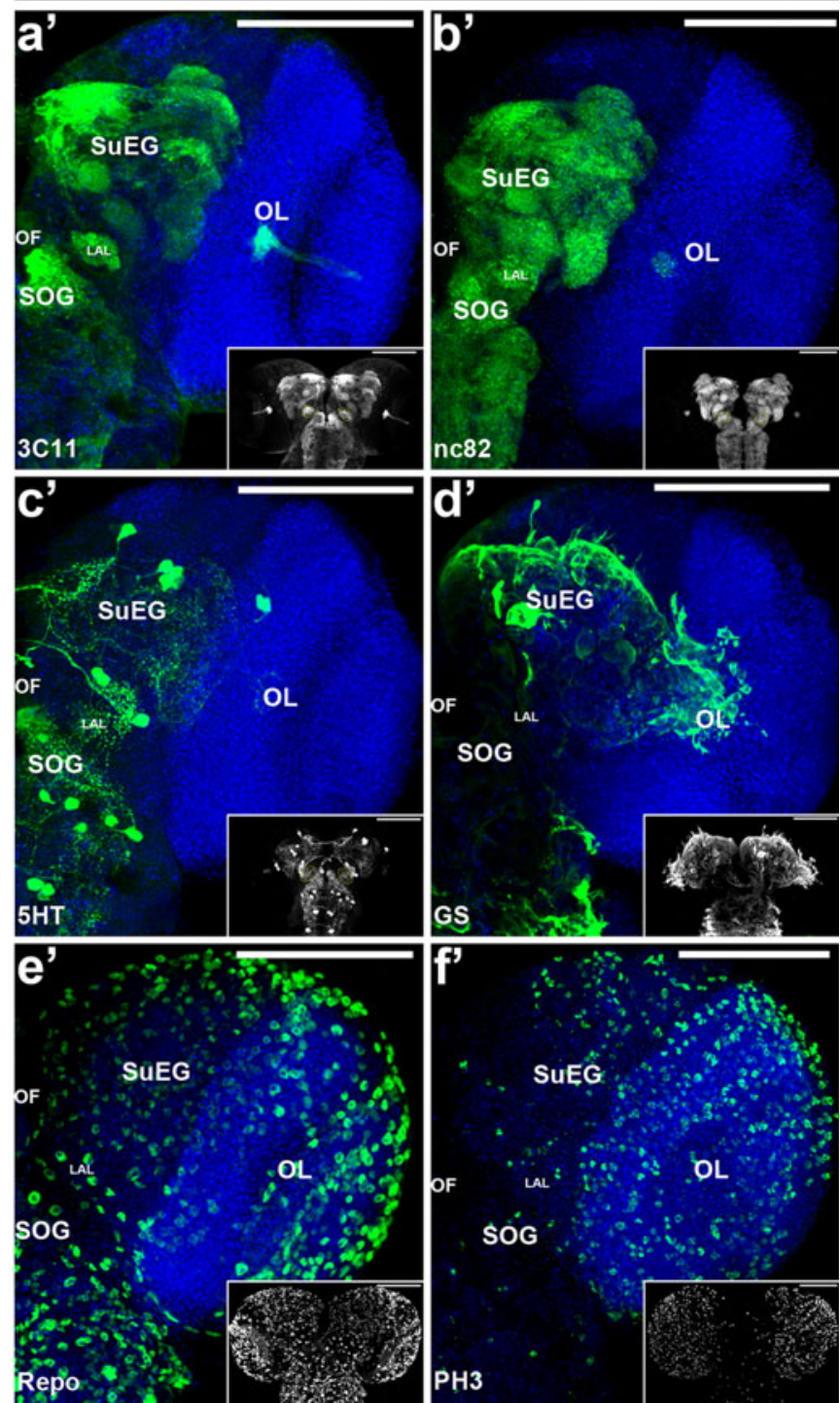

immunolabeling (d, $\mathbf{d}^{\prime}$ and $\left.\mathbf{e}, \mathbf{e}^{\prime}\right)$ labels glia that are associated with the cortical regions and the neuropil regions of the. Mitotic activity throughout the larval brain is indicated by anti-PH3 immunostaining $\left(\mathbf{f}, \mathbf{f}^{\prime}\right)$. $L A L$ larval antennal lobe, $O F$ esophageous foramen, $O L$ optic lobe, $S O G$ subesophageal ganglion, $S u E G$ supraesophageal ganglion. Yellow arrowhead indicates the connector between SuEG and SOG in Aedes brain. Scale bar $100 \mu \mathrm{m}$. The label on the left bottom of each image corresponds to the antibody represented

of the brain in mosquitoes and fruit fly (Fig. 1b, b'). This antibody also reveals a major difference in the organization of larval optic lobes in both the species and suggests that the mosquito possesses optic lobe structures at the larval stage that are more developed than its fruit fly counterpart. Anti-tubulin (tyrosinylated) immunolabeling strongly labels the cortical regions of the brain in both the insects, which comprise numerous cell bodies that project processes towards more central regions (Fig. 1c, $\mathrm{c}^{\prime}$ ). These central 


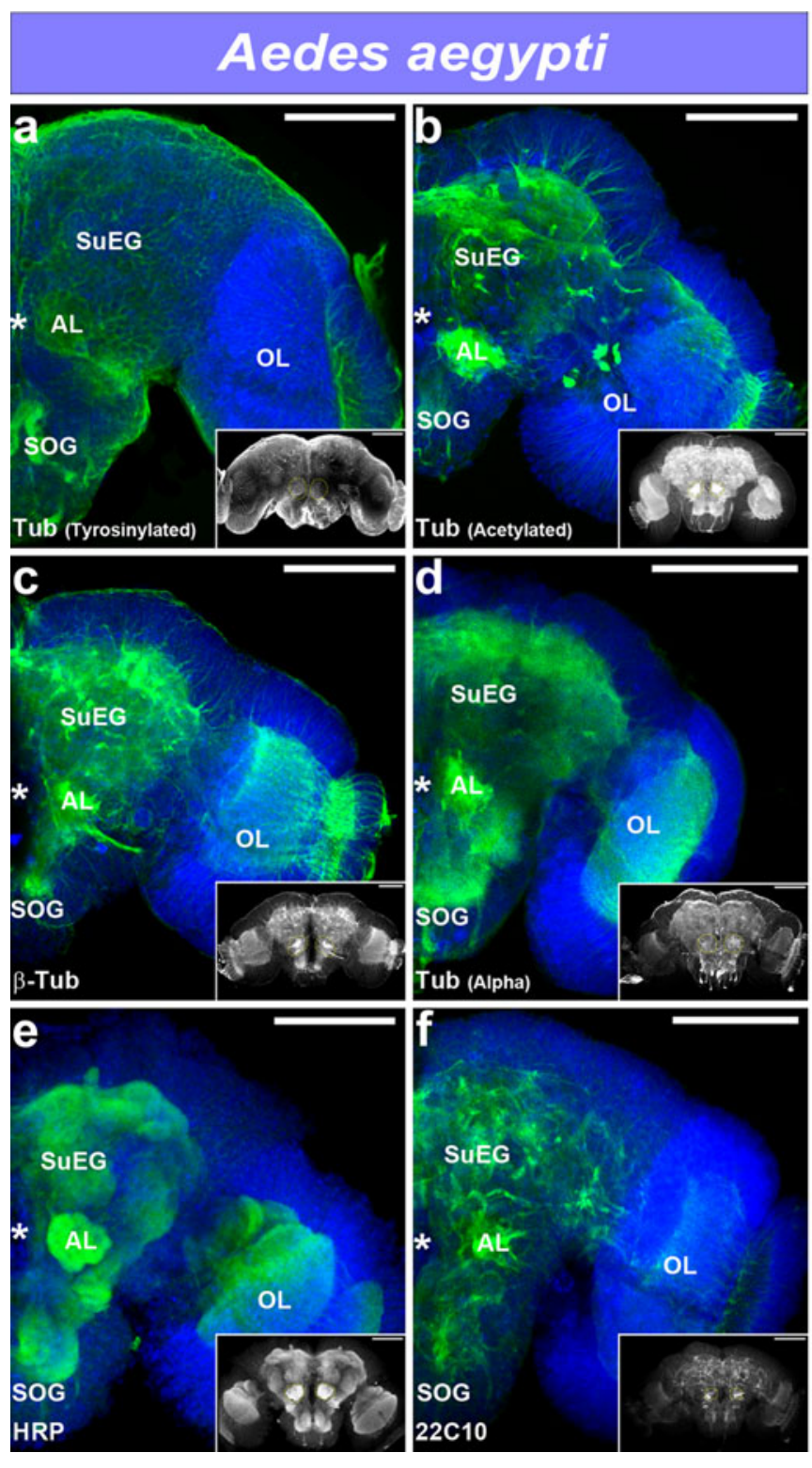

Fig. 3 Cross-reactivity of antibodies against the pupal ( $24 \mathrm{~h} \mathrm{APF)} \mathrm{brain} \mathrm{of}$ A. aegypti and a comparison with $45-50 \mathrm{~h}$ APF fruit fly pupal brains. Cortical and neuropil regions are labeled by anti-tubulin antibodies (a-d and $\mathbf{a}^{\prime}-\mathbf{d}^{\prime}$ ). A similar but more detailed labeling is seen in anti-HRP immunostained preparations (e) in case of mosquitoes and in fruitflies this antibody seems to mark nonspecifically the entire brain (e'); while anti-

regions of the brain are more prominently immunolabeled by anti- $\beta$ tubulin and anti- $\alpha$ tubulin antibodies; they correspond to the neuropil domains of the supraesophageal, subesophageal and optic lobe ganglia (Fig. 1d, d', e, e'). In addition, these antibodies reveal the detailed architecture of the developing optic lobe (Fig. 1d', ef ) of the fruit fly. Moreover, anti- $\beta$ tubulin immunolabeling of the larval brain demarcates what appear to be the axon tracts of secondary neuron lineages in both the mosquito and the fruit fly (Fig. 1d, d'). This immunolabeling also highlights clusters of cell bodies that give rise to these axon tracts and

\section{Drosophila melanogaster}
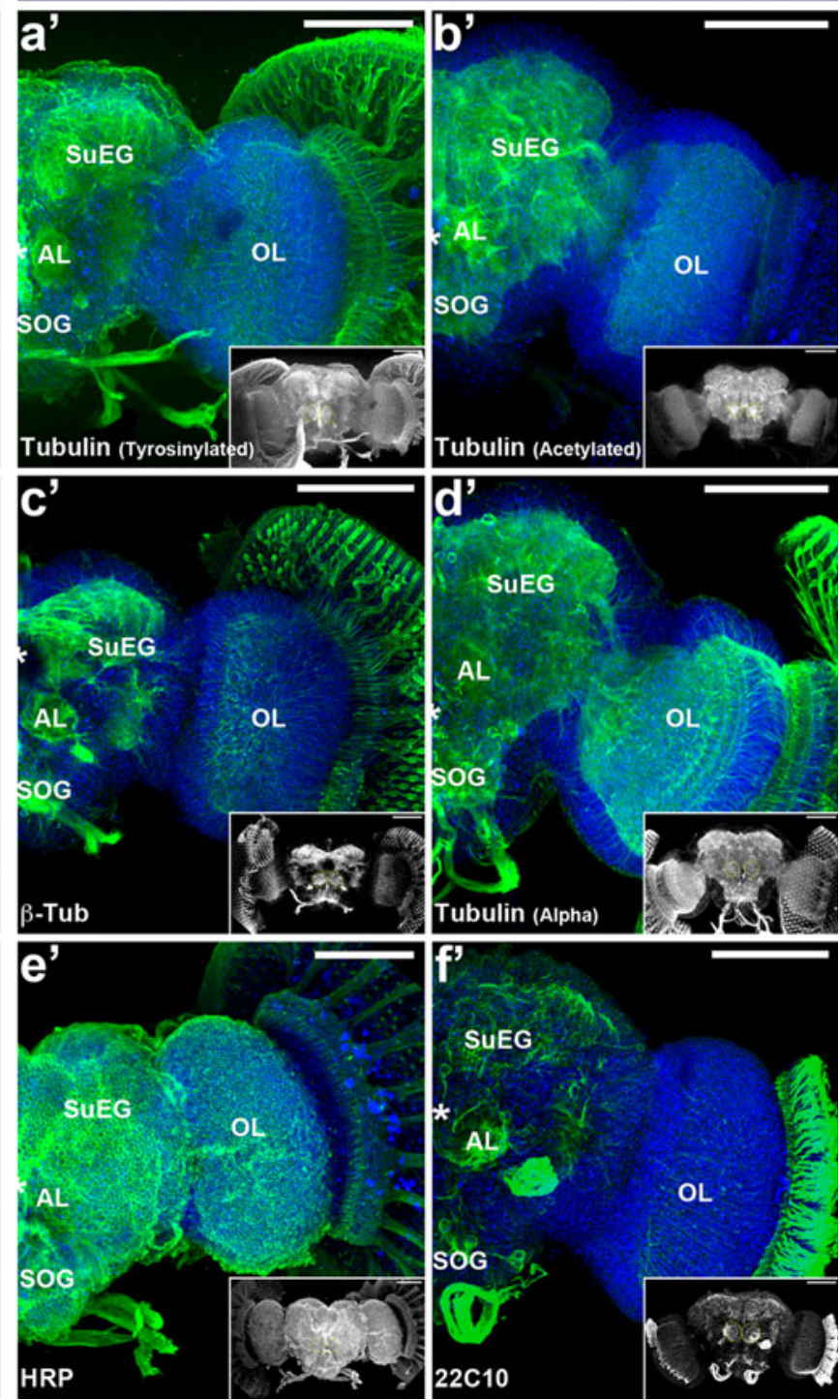

$22 \mathrm{C} 10$ antibody labels more discrete axon tracts and fascicles in the brain rather than compact neuropil domains $\left(\mathbf{f}, \mathbf{f}^{\prime}\right) . A L$ antennal lobe, $O L$ optic lobe, $S O G$ subesophageal ganglion, $S u E G$ supraesophageal ganglion; asterisk region of oesophageous foramen. Yellow dots in the inset highlight the antennal lobe in all the cases. Scale bar $100 \mu \mathrm{m}$. The label on the left bottom of each image corresponds to the antibody represented

hence may correspond to lineages in the mosquito that are comparable to those in Drosophila (Supp. Fig. 1d). Anti$22 \mathrm{C} 10$ immunostaining in the mosquito intensely labels specific neuropil regions and weakly labels cortical regions (Fig. 1f); in the fruit fly, the neuronal tracts in the optic lobe as well as in the cortical and the neuropilar regions are prominently labeled by this antibody (Fig. 1f').

More specific information on the organization of the neuropil domains in the central brain is obtained by anti$3 \mathrm{C} 11$ and anti-nc82 immunostaining. These immunolabels reveal a complex compartment-like organization in the neuro- 


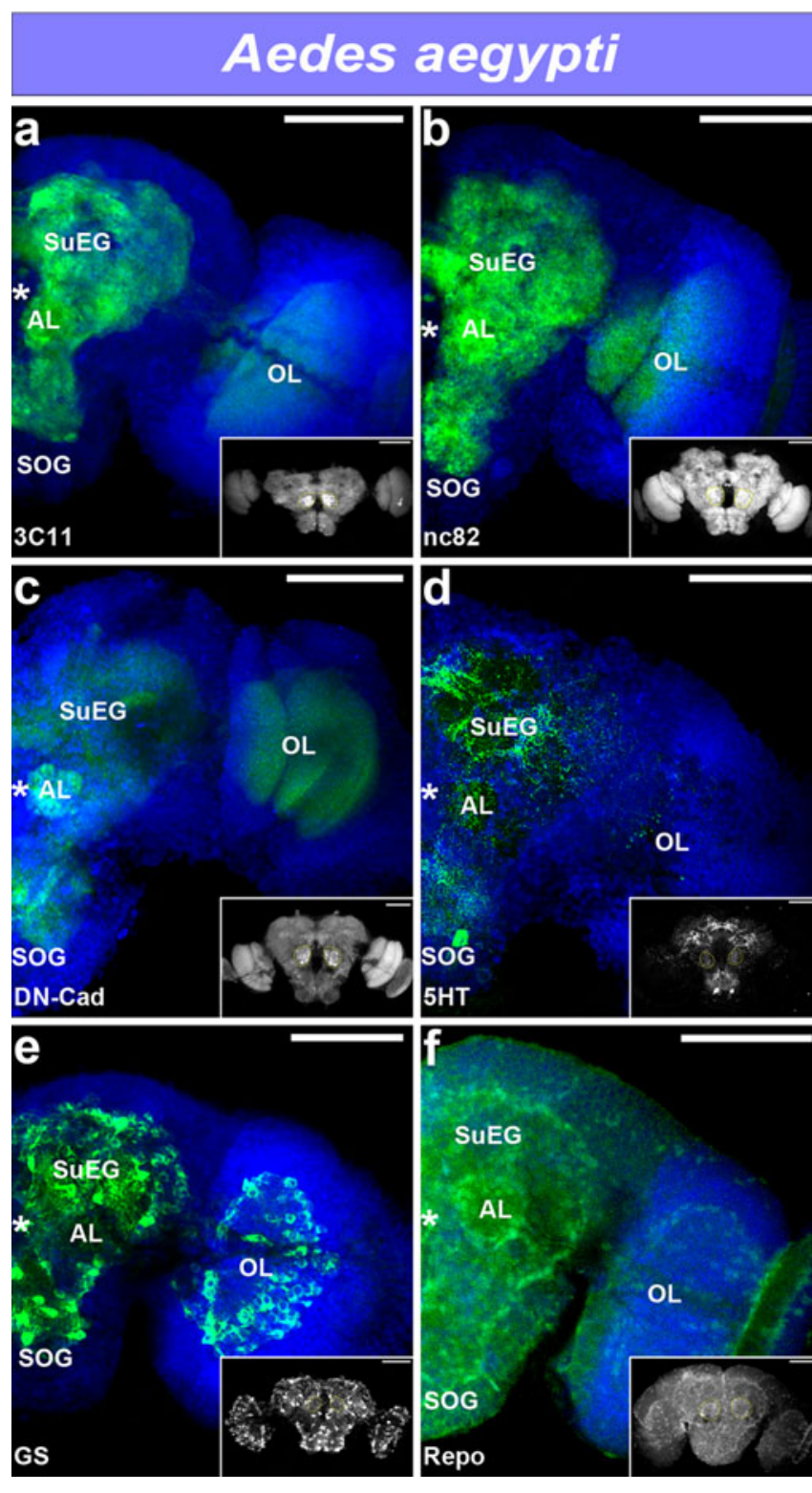

Fig. 4 Cross-reactivity of antibodies against the pupal (24 h APF) brain of A. aegypti and a comparison with $45-50 \mathrm{~h}$ APF fruit fly pupal brains. More precise information on the organization of the neuropils is obtained by anti-3 C11 (a, $\left.\mathbf{a}^{\prime}\right)$, anti-nc82 (b, $\left.\mathbf{b}^{\prime}\right)$ and anti-N-Cad immunostaining $\left(\mathbf{c}, \mathbf{c}^{\prime}\right)$. Whereas a restricted regionalized labeling is seen in anti-5HT immunostained pupal brains $\left(\mathbf{d}, \mathbf{d}^{\prime}\right)$. Glial cell bodies

pil of the supraesophageal and subesophageal ganglia of the mosquito (Fig. 2a, b). A similar pattern is observed in the fruit fly. However, the fruit fly seems to have somewhat more complex compartmentalized architecture when compared with mosquito larval brain (Fig. 2a', b'). Highly restricted labeling in the neuropil is shown by anti-5HT immunostaining, which reveals the 5HT immunoreactive cell bodies as well as their projections in the neuropil, commissures, and connectives of the supraesophageal and subesophageal ganglia (Fig. 2c, $\mathrm{c}^{\prime}$ ) in both the mosquito and the fruit fly. Numerous glial cells that are associated with the cortical

\section{Drosophila melanogaster}
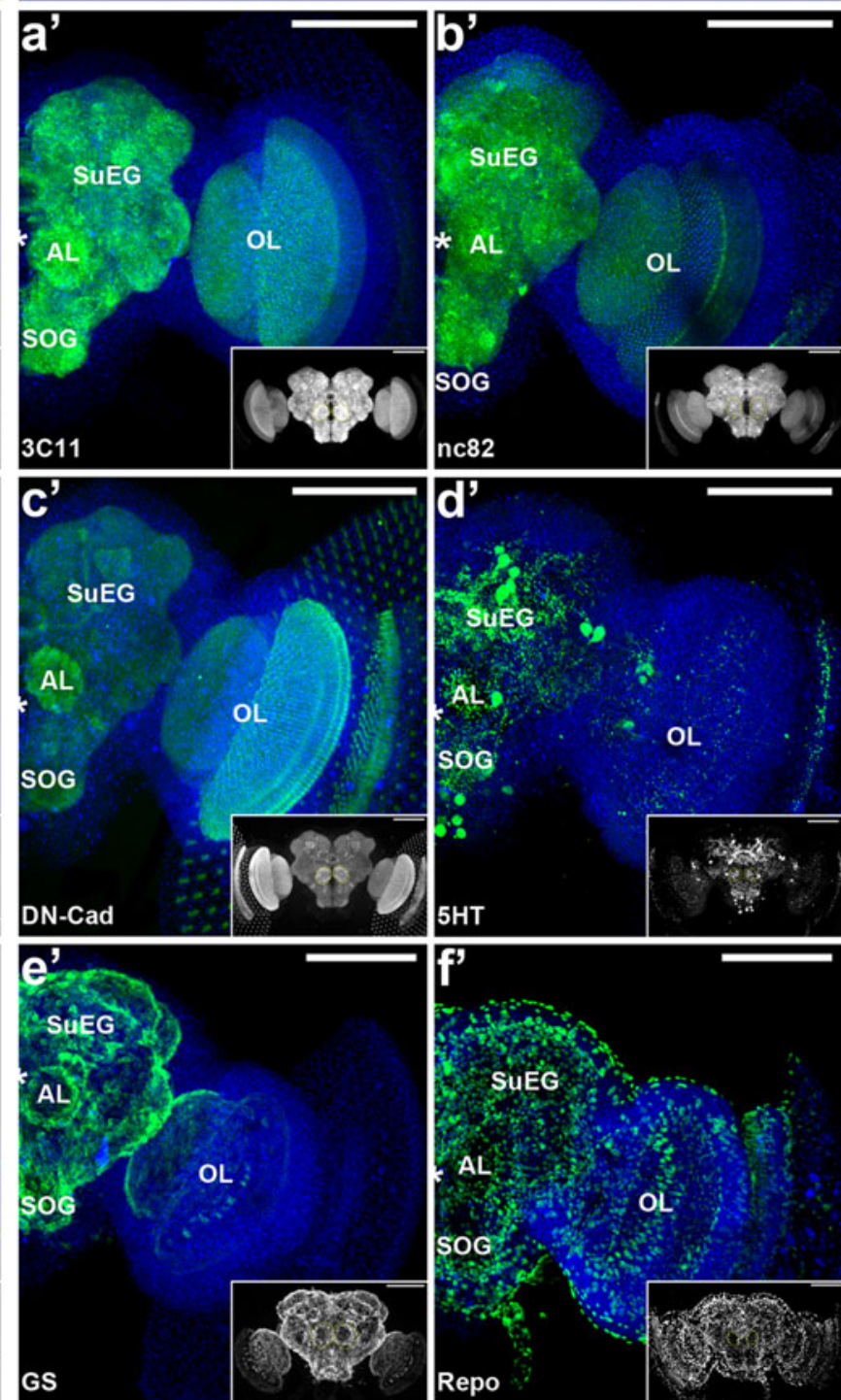

are immunolabeled with anti-GS antibody $\left(\mathbf{e}, \mathbf{e}^{\prime}\right)$ and as well anti-repo immunolabeling $\left(\mathbf{f}, \mathbf{f}^{\prime}\right) . A L$ antennal lobe, $O L$ optic lobe, $S O G$ subesophageal ganglion, $S u E G$ supraesophageal ganglion; asterisk region of oesophageous foramen. Yellow dots in the inset highlight the antennal lobe in all the cases. Scale bar $100 \mu \mathrm{m}$. The label on the left bottom of each image corresponds to the antibody represented

regions and the neuropil regions of the mosquito brain are revealed by anti-GS immunolabeling and also by anti-Repo immunolabeling albeit less clearly (Fig. 2d, e). Application of the same antibodies to the fruit fly brain also labels glial cells (Fig. 2d', e'). Evidence for mitotic activity throughout the larval brain in mosquito and fruit fly is provided by anti-PH3 immunostaining (Fig. 2f, $\mathrm{f}^{\prime}$ ). Anti-PH3 immunolabeling is seen in the optic lobe, supraesophageal, and subesophageal ganglia; immunopositive cell bodies are located both in cortical and in neuropil regions, suggesting that both neuronal cells and glial cells are being generated. 


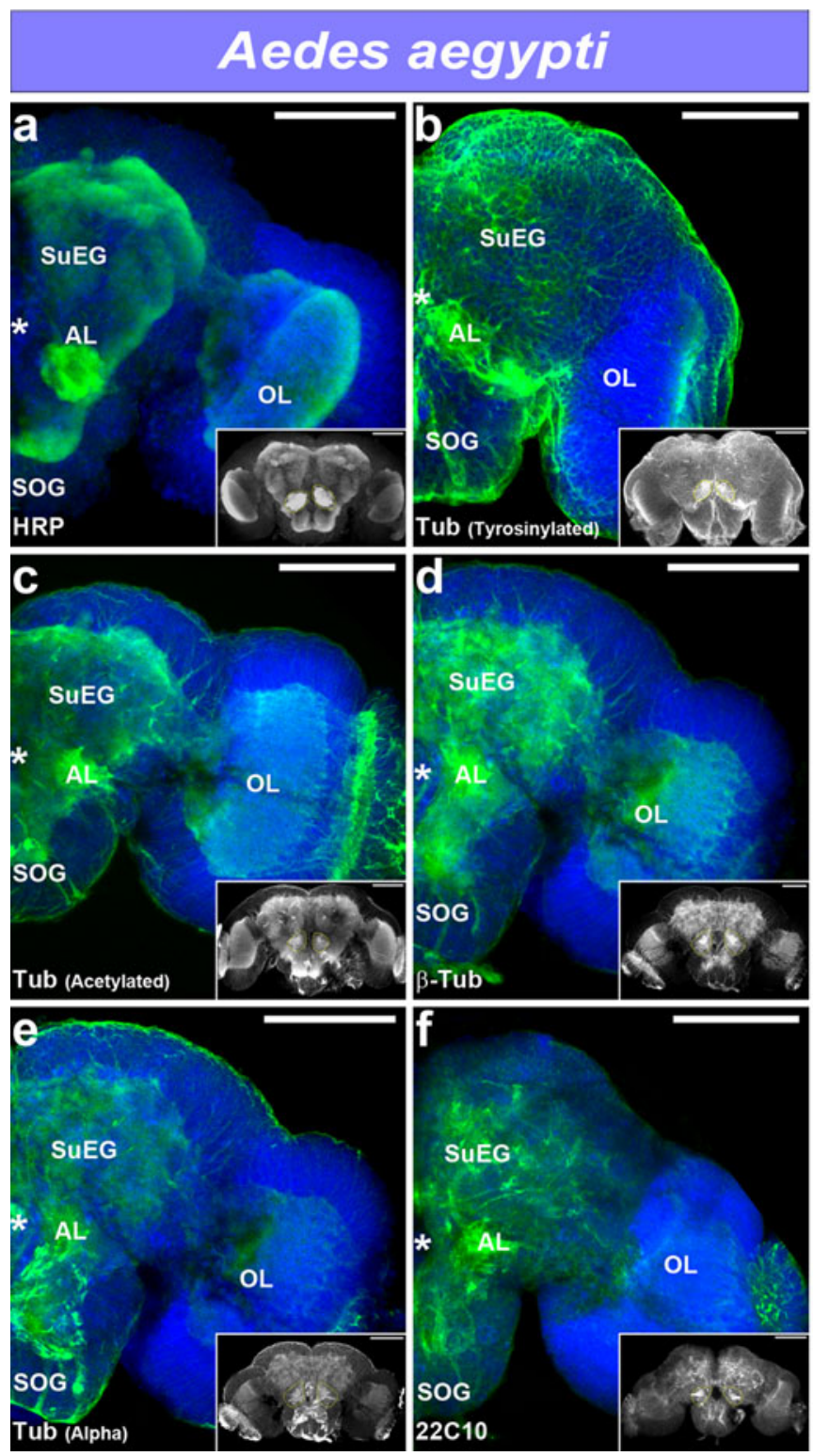

Fig. 5 Cross-reactivity of antibodies against the adult brain of $A$. aegypti along a comparison with adults of frutifly $D$. melanogaster. Labeling by anti-HRP (a) and anti-tubulin antibodies (b-e and $\left.\mathbf{b}^{\prime}-\mathbf{e}^{\prime}\right)$ reveals general features of the adult brain. More specific immunolabeling of neuropil regions with anti-22 $\mathrm{C} 10\left(\mathbf{f}, \mathbf{f}^{\prime}\right)$ also reveals features that are very similar

Organization of the developing brain in the Aedes pupal stage

In general, the anatomical features of the pupal brains of mosquito and fruit fly are much more similar than those of the corresponding larval brains. This is clearly manifested by immunolabeling with anti-tubulin antibodies, which provide information about the overall features of the developing brain of the pupal stage of the mosquito ( $24 \mathrm{~h} \mathrm{APF}$ ) and the fruit fly (45-50 h APF). Cortical regions of the pupal brain are labeled by anti-tubulin (tyrosinylated) immunostaining and neuropil regions are labeled by anti-tubulin (acetylated), anti- $\beta$ tubulin

\section{Drosophila melanogaster}
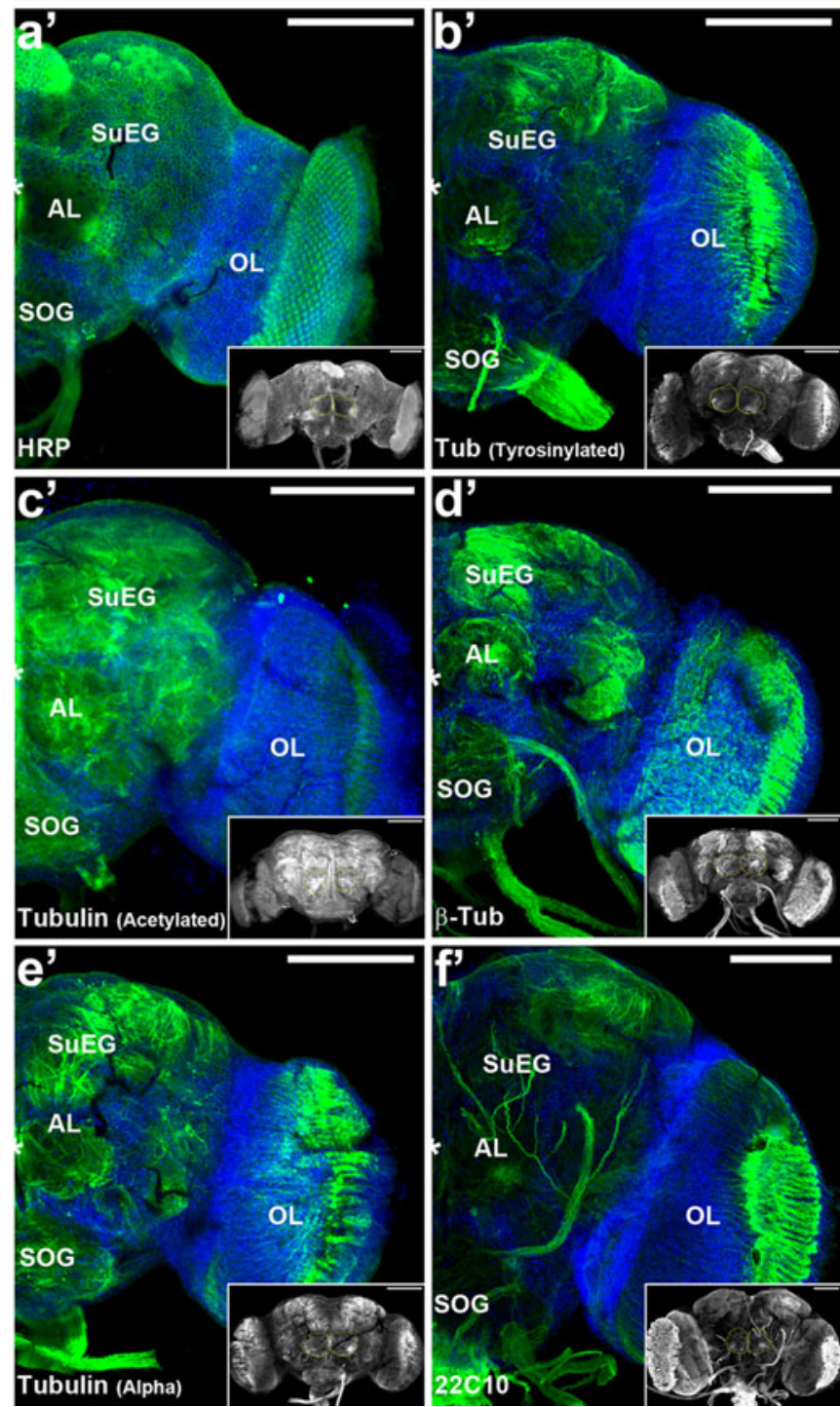

to the pupal brain. $A L$ antennal lobe, $O L$ optic lobe, $S O G$ subesophageal ganglion, SuEG supraesophageal ganglion; asterisk region of oesophageous foramen. Yellow dots in the inset highlight the antennal lobe in all the cases. Scale bar $100 \mu \mathrm{m}$. The label on the left bottom of each image corresponds to the antibody represented

and anti- $\alpha$ tubulin immunostaining (Fig. 3a-d, $a^{\prime}-d^{\prime}$ ). In terms of general neuroanatomy, all of these anti-tubulin antibodies reveal a more compact organization of the mosquito pupal brain as compared to the mosquito larval brain. Thus, in the mosquito pupal brain, the subesophageal ganglion has fused with the supraesophageal ganglion and optic lobes (Fig. 3a, b). Moreover prominent and highly complex compartments have developed in the supraesophageal ganglion, the (fused) subesophageal ganglion and in the optic lobes (Fig. 3b, c). Among these prominent labeled neuropil structures are the bilateral pupal antennal lobes (highlighted by yellow dots in the inset and Supp. Figs. 3 

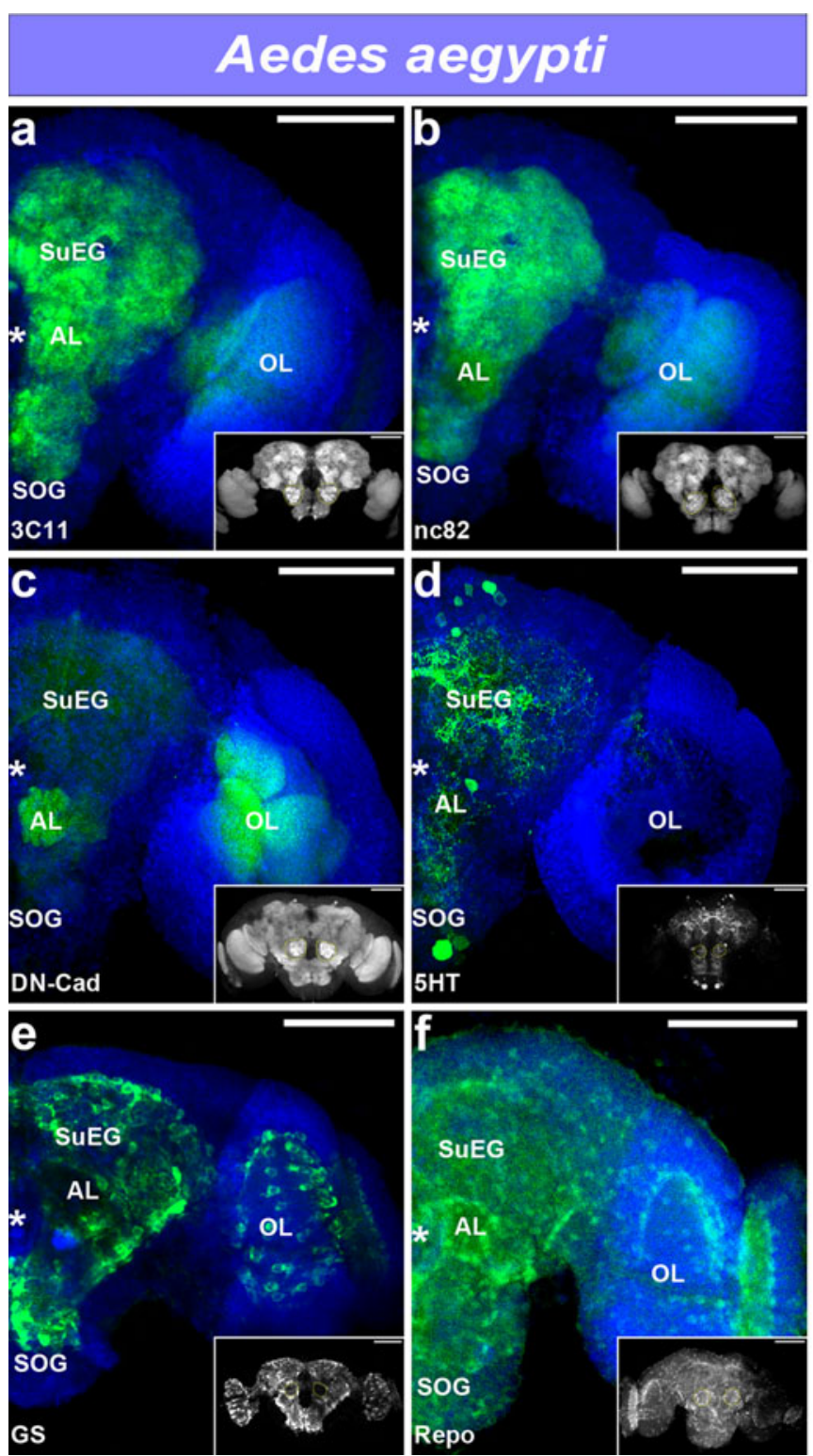

Fig. 6 Cross-reactivity of antibodies against the adult brain of $A$. aegypti along a comparison with adults of frutifly D. melanogaster. anti-3 C11 (a, $\left.\mathbf{a}^{\prime}\right)$, anti-nc82 (b, b') and anti-DN Cadherin (c, $\left.\mathbf{c}^{\prime}\right)$ reveals more precise information on the neuropilar organization in both the insects. Immunostaining with the anti-5HT $\left(\mathbf{d}, \mathbf{d}^{\prime}\right)$ antibody, anti-GS (e, $\left.\mathbf{e}^{\prime}\right)$ and anti-Repo (f, $\left.\mathbf{f}^{\prime}\right)$ antibodies labels serotonergic

and 4) that are located lateral to the small foramen (white asterisk in Figs. 3 and 4). Comparable labeling of the pupal brain neuropil in mosquitos is seen in anti-HRP immunostained preparations. Indeed, in these preparations the neuropil compartments are more defined and antennal lobes as well as subdivisions in the optic lobes are clearly manifest (Fig. 3e). In contrast, anti-HRP immunolabeling of the fruit fly pupal brain reveals a more unspecific marking of the entire cortical region of the pupal brain without any specific labeling of neuropil structures (Fig. 3e'). Finally, labeling with the anti-22 $\mathrm{C} 10$ antibody reveals more discrete axon

\section{Drosophila melanogaster}
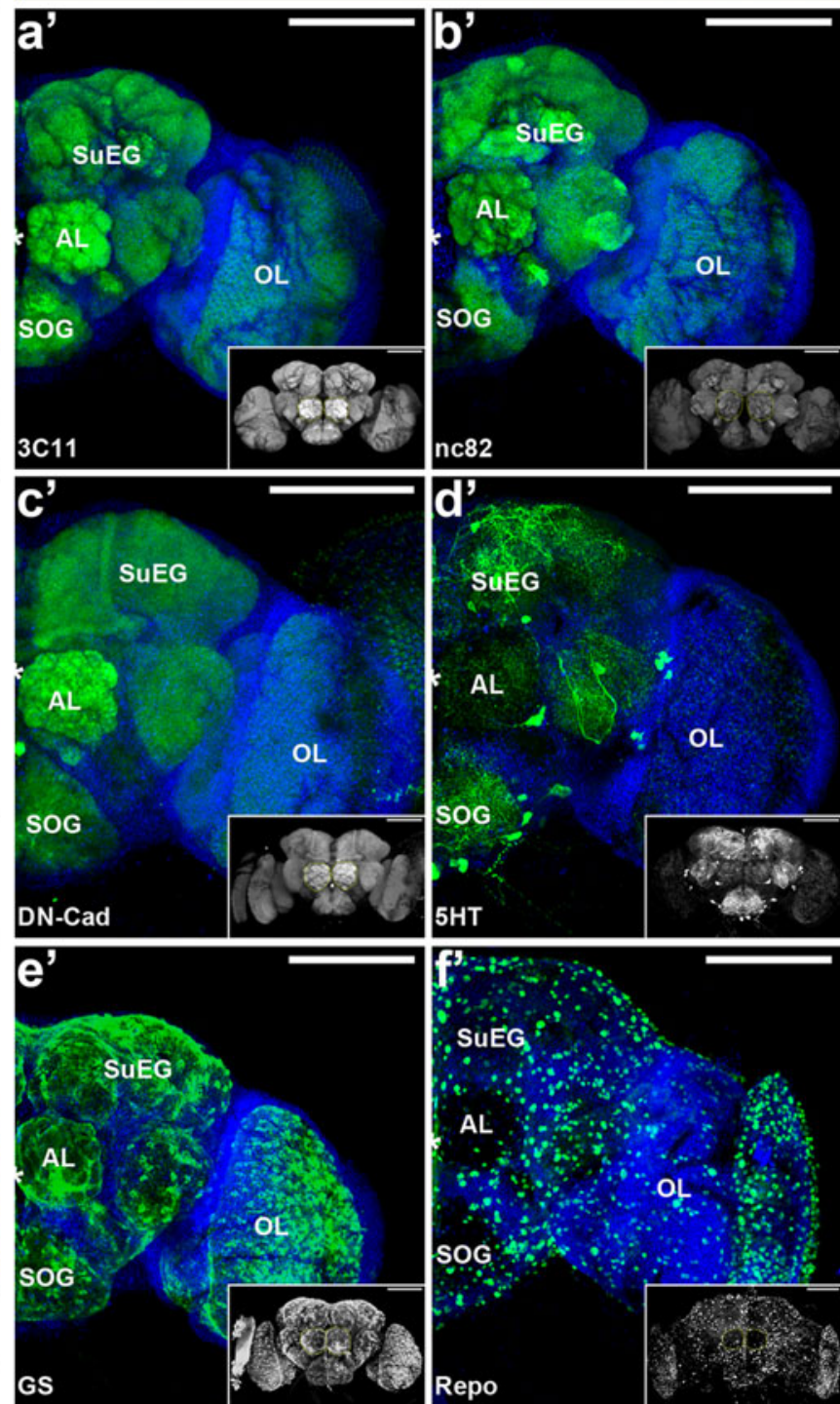

GS

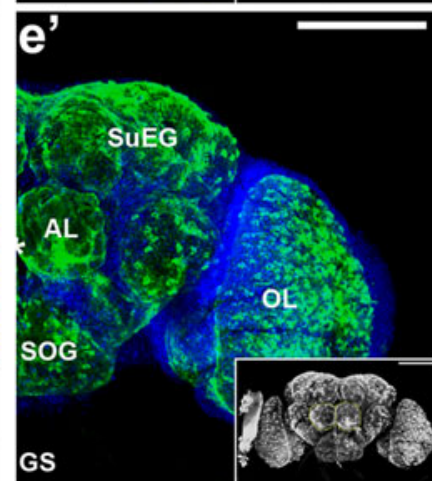

Repo

neurons and glial cells respectively in the adult brain. $A L$ antennal lobe, $O L$ optic lobe, $S O G$ subesophageal ganglion, $S U E G$ supraesophageal ganglion; asterisk region of oesophageous foramen. Yellow dots in the inset highlight the antennal lobe in all the cases. Scale bar $100 \mu \mathrm{m}$. The label on the left bottom of each image corresponds to the antibody represented

tracts and fascicles rather than compact neuropil domains in the brain of both mosquito and fruit fly (Fig. 3f, f').

Additional information on the organization of the neuropil domains in the pupal brain is obtained by anti3 C11, anti-nc82 and anti-DN-Cad immunostaining (Fig. 4a-c). Labeling with these antibodies reveals the paired antennal lobes, an unpaired midline neuropil corresponding to the central complex, and a subdivision of the optic lobes into a peripheral lamina, a large medial medulla and a proximal lobula. Applying these antibodies to the fruit fly brain also reveals similar features 
(Fig. $4 \mathrm{a}^{\prime}-\mathrm{c}^{\prime}$ ). These antibodies further underscore the remarkable similarity in the anatomical organization of the pupal brains in both mosquito and fruit fly observed earlier. Remarkably restricted regionalized labeling is seen in anti5HT immunostained pupal brains of mosquito and fruit fly, in which single neuronal cells innervating the antennal lobe and a small set of neurons in the subesophageal ganglion are immunolabeled; neuronal processes in several other regions of the neuropil as well as in a restricted region in the central complex are also 5HT immunopositive (Fig. 4d, d'). In both insects, glial cell bodies immunolabeled with anti-GS are associated with the neuropil in all regions of the pupal brain (Fig. 4e, e'). Moreover, some evidence for glial cells associated with cortical regions (as well as neuropil regions) is revealed by anti-Repo immunolabeling (Fig. 4f, $\mathrm{f}^{\prime}$ ). Interestingly, and in contrast to the larval brain, evidence for mitotic activity is completely lacking at $24 \mathrm{~h} \mathrm{APF}$ in mosquito and at $45-50 \mathrm{~h} \mathrm{APF}$ in fruit fly; anti-PH3 immunostaining does not reveal any immunopositive cells in the pupal brains of mosquito and fruit fly (data not shown).

\section{Organization of the adult brain in Aedes}

Immunolabeling of the adult mosquito and fruit fly brain reveals features that are very similar to those observed in immunolabeled pupal brains (at $24 \mathrm{~h} \mathrm{APF}$ for mosquitoes/45-50 h APF for fruitflies). Thus, labeling by anti-HRP and anti-tubulin antibodies reveals general features of the adult brain that are directly comparable to those observed in the pupal brains of mosquitoes (Fig. 5a-e) and fruitflies (Fig. 5a'-e'). Labeling of the cortical regions of the brain and of the distinct neuropil regions of the brain is very similar in adult and pupal brains immunostained with these antibodies. Supraesophageal and subesophageal ganglia are fused, prominent, and complex neuropilar regions are present in these ganglia, and the optic lobes are subdivided into lamina, medulla, and lobula. More specific immunolabeling of neuropil regions with anti-22 C10, anti-3 C11, anti-nc82, and anti-DN cadherin also reveals features, which are very similar in the adult and pupal brain (Figs. 5f, $\mathrm{f}^{\prime}$ and $6 \mathrm{a}-\mathrm{c}, \mathrm{a}^{\prime}-\mathrm{c}^{\prime}$ ). Among these are the paired antennal lobes (highlighted by yellow dots in the inset and Supp. Figs. 5 and 6) and the unpaired midline neuropil of the central complex, both of which are prominently labeled. Similarly, immunostaining with the anti-5HT (Fig. 6d, d') antibody as well as with the glial-specific anti-GS and anti-Repo antibodies labels directly comparable cells in the adult and in the pupal brain (Fig. 6e-f, $\left.\mathrm{e}^{\prime}-\mathrm{f}^{\prime}\right)$. Finally, anti-PH3 immunostaining indicates that mitotic activity is also absent in the adult brain (data not shown).
Development of specific brain neuropils in Aedes

In addition to the characterization of the general features of the brain at different developmental stages, immunolabeling with cross-reacting antibodies also allows detailed analysis of the development of specific components of the brain in Aedes. Here, we use two of these antibodies, anti-3 C11 and anti-5HT, to characterize the development of (a) the optic lobe neuropil, (b) the subesophageal ganglion neuropil, and (c) the serotonergic cell system in the larval, pupal, and adult subesophageal ganglion of the Aedes brain.

As documented above, the Aedes brain comprises distinct optic lobe neuropils at larval, pupal, and adult stages. To document the spatial and temporal development of this neuropil in more detail, we use anti-3 C11 immunolabeling in combination with laser confocal optical sectioning in these three stages. In the larva, the optic lobe neuropil is clearly subdivided into three domains, which correspond to the lamina, medulla, and lobula of the larval brain (Fig. 7a-a"). The neuropil of the (weakly labeled) lamina and the neuropil of the distinctly larger medulla do not yet show any obvious subdivisions in immunostained preparation at this stage. In contrast, the lobula neuropil, although significantly smaller than the medulla neuropil, does appear to manifest two layer-like subdivisions. In the pupa, all three neuropils have grown markedly in size (Fig. 7b-b"). The medulla neuropil is still the largest of the three; it is now clearly subdivided into an outer neuropil ("outer medulla") and an inner neuropil ("inner medulla"). The subdivision of the lobula into an inner and an outer layer, which is already apparent in the larva, remains distinctly visible. The three optic lobes of the adult brain are very similar to the optic lobes of the pupal brain (Fig. $7 \mathrm{c}-\mathrm{c}^{\prime \prime}$ ). Thus, adult and pupal lamina, medulla, and lobula are almost identical in size and in terms of their internal subdivisions; medulla and lobula of the adult brain are clearly subdivided into outer and inner neuropil strata.

In Aedes, the subesophageal ganglion of the larva is distinctly separated from the supraesophageal ganglion by the large oesophageal foramen. In contrast in pupal and adult stages, the subesophageal ganglion is fused with the supraesophageal ganglion and the esophageal foramen is markedly reduced in size (see above). In addition, a remarkable increase in the size and complexity of the subesophageal neuropil is manifest in larval versus pupal brains. This dramatic increase is clearly evident in the anti3 C11 immunolabeled neuropils of the subesophageal ganglia (Fig. 7d-d", e-e"). In all three optical sections shown, the pupal neuropil is larger and characterized by numerous subcompartment-like structures that are not present in the larval neuropil. In contrast, the neuropils of the pupal and adult subesophageal ganglia are quite similar both in size and in terms of their internal subdivisions 

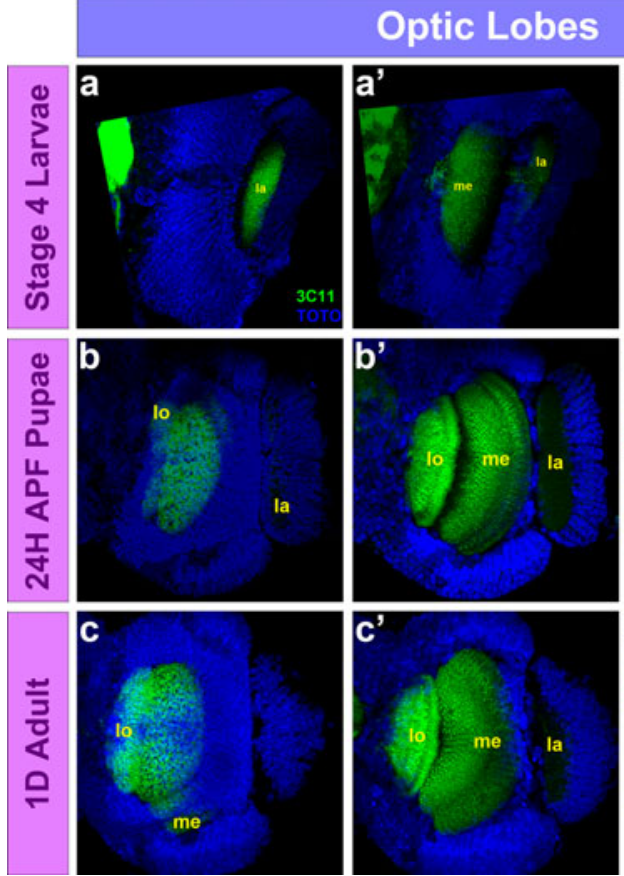

Fig. 7 Spatial and temporal development of the optic lobe and the subesophageal ganglion neuropil in A. aegypti brain as revealed by anti$3 \mathrm{C} 11$ antibody labeling. In the larva, the optic lobe neuropil can be subdivided into lamina (la), medulla (me) and lobula $\left(l o ; \mathbf{a}-\mathbf{a}^{\prime \prime}\right)$. In the pupa, all the three neuropils have grown markedly in size $\left(\mathbf{b}-\mathbf{b}^{\prime \prime}\right)$ and has a very similar anatomy as in the adult $\left(\mathbf{c}-\mathbf{c}^{\prime \prime}\right)$. In case of the

(Fig. 7e-e", $\mathrm{f}-\mathrm{f}^{\prime \prime}$ ). This suggests that significant growth/ restructuring of the subesophageal neuropil occurs during development from the larva to the pupa.

As in other insect brains, a small set of serotoninergic neurons is found in the supraesophageal and subesophageal ganglia of Aedes, and the development of these neurons can be characterized by anti-5HT immunolabeling (see above). By focusing on the subset of serotoninergic neurons in the subesophageal ganglia, it is possible to study the differentiation of these neurons at the level of single cells, and at the same time document the dramatic increase in neuropil complexity that occurs in this ganglion during development from the larva to the pupa. In the larval brain, two groups of 5HT-immunoreactive cells, one located anteriorly and one posteriorly, each comprising two to three cells per group is present in each hemiganglion (Fig. 8a-a'). These cells give rise to 5HT-immunoreactive fibers in the relatively small subesophageal neuropil of the larva. In the pupal brain, three groups of immunoreactive cells are visible in each hemiganglion, one anterior group, one medial group and one posterior group, and each of these groups comprises three cells (Fig. 8b- $b^{\prime}$ ). This increase in the number of 5HTimmunoreactive cells is reflected by the increase in density of 5HT-immunoreactive fibers in the significantly enlarged subesophageal neuropil of the pupa. The 5HT-immunoreactive cells in the adult brain are very similar to those in the pupal
Sub Oesophageal Ganglion
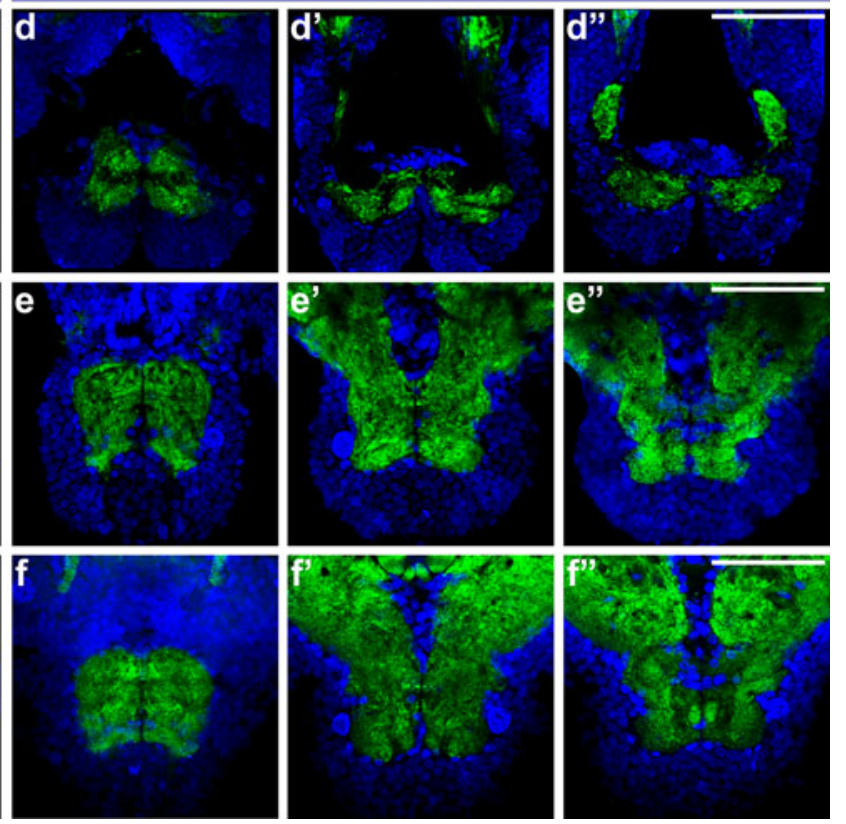

subesophageal ganglion, the larval subesophageal ganglion is distinctly separated from the supraesophageal ganglion by the large esophageal foramen $\left(\mathbf{d}-\mathbf{d}^{\prime \prime}\right)$. While this neuropil in pupae $\left(\mathbf{e}-\mathbf{e}^{\prime \prime}\right)$ and adult $\left(\mathbf{f}-\mathbf{f}^{\prime \prime}\right)$ shows a remarkable increase in the size and complexity suggesting a significant growth/restructuring of the subesophageal neuropil during development from the larva to the pupa. Scale bar $50 \mu \mathrm{m}$

brain (Fig. $8 \mathrm{c}-\mathrm{c}^{\prime}$ ). The only apparent difference between pupa and adult is an increase in the density of immunoreactive fibers in the neuropil.

\section{Discussion}

In model insects such as Drosophila, antibodies have been used as powerful tools for understanding the development of the nervous system at the molecular, cellular, and histological level. In this report, we screen a number of these Drosophila antibodies for cross-reactivity in the major disease vector, the yellow fever mosquito $A$. aegypti. The suite of 13 (Table 1) cross-reacting antibodies identified here should be useful for subsequent studies of the developing mosquito nervous system and, since all of these antibodies also label the adult mosquito nervous system, should also contribute to further understanding of the organization and anatomy of the adult mosquito brain. Among these cross-reacting antibodies are antibodies that label the entire nervous system (pan-neuronal) exemplified by anti-HRP and anti-tubulin antibodies, antibodies that label more restricted components of the nervous system such as the neuropil exemplified by the anti-nc 82 and anti$3 \mathrm{C} 11$ antibodies, and antibodies that label highly specific subcomponents of the nervous system such as small sets of neurons exemplified by the anti-5HT antibody. Interesting- 


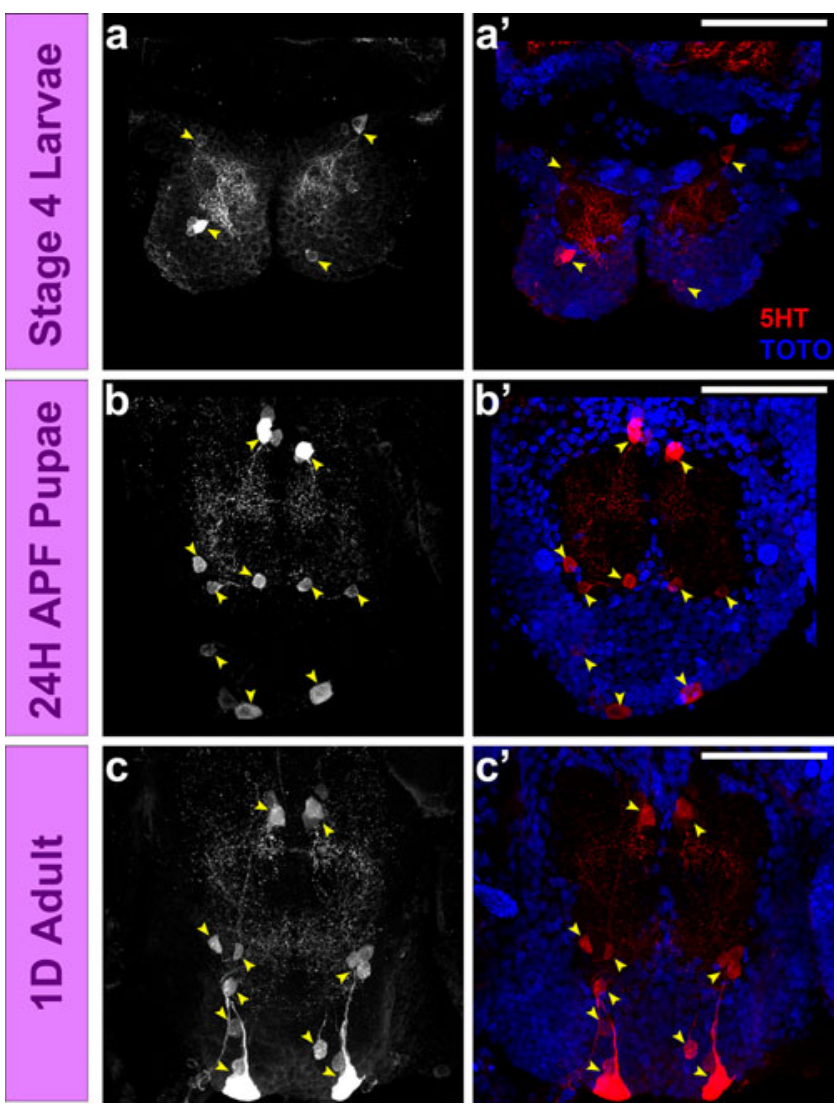

Fig. 8 Patterns of serotonergic innervations of the subesophageal ganglion in a developing $A$. aegypti brain. In the larval subesophageal ganglion (a-a'), two groups of 5HT-immunoreactive cells can be identified (white/yellow arrow heads) per hemiganglion. Whereas in the pupal $\left(\mathbf{b}-\mathbf{b}^{\prime}\right)$ and adult $\left(\mathbf{c}-\mathbf{c}^{\prime}\right)$ brains three groups of 5HT immunoreactive cells can be identified (whitelyellow arrow heads) in each hemiganglion. Scale bar $50 \mu \mathrm{m}$

ly, for the antibodies studied here, the general type of labeling (pan-neuronal, more restricted, and highly specific) manifested by any given antibody is comparable throughout all stages of neuronal development. This diversity of immunolabeling types should add to the utility of the suite of cross-reacting antibody in further studies.

The immunohistological analysis presented here reveals two striking features of brain organization in the different life cycle stages of Aedes. The first is the remarkable difference in the organization of the brain in the larva versus the pupa. In the larval brain, the supraesophageal and subesophageal ganglia are distinct and unfused, and are interconnected by prominent connectives, which project around an extremely large oesophageal foramen. Moreover, the brain neuropil is reduced in size and complexity, and it manifests few internal subdivisions. In contrast, in the pupal brain, the supraesophageal and subesophageal ganglia are fused and the oesophageal foramen is strongly reduced. The brain neuropil is dramatically increased in size and complexity, and shows extensive compartment-like subdivisions such as antennal lobes, central complex, and the subdivisions manifest in the optic lobes. Given that the larval stage studied here is the last of the four larval instars, the striking difference between the brain at this larval stage and at the pupal stage ( $24 \mathrm{~h} \mathrm{APF}$ ) must be generated in the space of approximately $24 \mathrm{~h}$, implying that dramatic growth and reorganization processes occur in this limited space in time. Moreover, since there is ample evidence of proliferative mitotic activity in the larval brain but none whatsoever in the pupal brain (24 h APF), much of this growth and reorganization is likely to occur in the very early stages of pupal development when proliferation might still be ongoing as reported for other holometabolous insects (Ishii et al. 2005; Mysore et al. 2010). The second striking feature in brain development is the remarkable similarity in the organization of the $24 \mathrm{~h}$ pupal brain and the adult brain. Indeed in most of the anatomical aspects revealed by our immunolabeling experiments, the pupal and the adult brain are virtually identical. Since the overall pupal development period is approximately $48 \mathrm{~h}$, this similarity in brain organization means that an "adult-like" organization of the brain is already attained within the first half of pupal development. Taken together these findings indicate that a number of critical developmental events must take place during the first day of pupal development. It will be important to investigate these events in more detail in future studies of brain development in Aedes.

Our developmental analysis reveals several remarkable similarities in brain development of Aedes versus Drosophila despite the 250 million years of divergent evolution that separate these two Dipteran insects. First, neural proliferative activity in the brain appears to occur primarily in the larval stage, whereas extensive neural growth and rearrangement takes place in the pupal stage likely reflecting process outgrowth and synapse formation in pupal stages. Second, the complex neuropils that are characteristic of the adult brain such as the antennal lobe, central complex, and three optic lobe neuropils are formed in the pupa in both insects. Third, the neuroanatomical organization of the corresponding brain neuropils at the histological level, and in some cases at the cellular level, is strikingly similar in the two Dipteran insects. Nevertheless, there are also prominent differences in brain development of Aedes as compared to Drosophila. Perhaps the most remarkable of these differences is that the larval stage of Aedes already has well-developed optic lobes whereas the optic lobes are present only as primordia in Drosophila (Hofbauer and Campos-Ortega 1990; Meinertzhagen and Hanson 1993; Sprecher et al. 2011). The presence of a well-developed optic lobe structures in the mosquito larvae is explained by its escape behavior from the air-water interface when threatened form above (Mellanby 1958; Olsson and Klowden 1998); a possible response to avoid predation. 
These larvae feed generally on the microbiota like algae in their natural environment; this may explain the relatively large size of foramen as compared to that of the Drosophila larva (or of the liquid-feeding adult Aedes). The large foramen, in turn, may also be responsible for the unfused nature of the supraesophageal and subesophageal ganglia in Aedes larva. This contrasts with Drosophila larval brains where both ganglia are fused into a compact larval brain. In both respects (optic lobe, foramen), we posit that specific ecological constraints of different lifestyles of the animals may be directly reflected in the development of their brains. Thus, mosquito larvae reside in small water bodies that are sunlit, turbid, temporary, and lack consistent supply of food (Merritt et al. 1992), while Drosophila larvae typically exist in a terrestrial environment with a reasonably constant supply of food. These differences in life styles of the larvae of these two species might result in different behavioral requirements that are reflected in differential developmental events during the construction of the larval brain.

A fundamental feature of brain development in Drosophila is the fact that a set of approximately 100 stem cell-like neuroblasts give rise to clonal units referred to as neuroblast lineages that comprise all of the neurons in the central brain (reviewed in Hartenstein et al. 2008; Spindler and Hartenstein 2010). In this process, the neuronal progeny of each neuroblast remain closely associated such that their cell bodies form discrete clusters and their axons fasciculate into a common axon tract. In contrast to the primary neurons that arise during embryogenesis, the majority of the neurons in the fly brain are generated postembryonically during the larval stage, and these so-called secondary, adult-specific neurons form secondary axon tracts in the larval brain. In consequence, each brain neuroblast generates a specific identifiable lineage of secondary neurons, which form a characteristic invariant secondary axon tract in the larval brain. During subsequent pupal stages, the secondary neurons in these lineages mature, the axons in the secondary neuron axon tracts form terminal arbors and undergo synaptogenesis giving rise to the circuitry of the adult brain. From this clonal standpoint, the neuroblast lineages are a fundamental "unit of projection" in the developing Drosophila brain (Larsen et al. 2009). Our analysis of the developing larval brain in Aedes suggests that similar neuroblast lineages and their corresponding secondary axon tracts might be salient feature of this dipteran too. In the larval mosquito brain, we find peripheral clusters of cell bodies that project characteristic axon tracts into the developing neuropil that are most clearly manifest in anti- $\beta$ tubulin immunolabeling. It will be important to analyze these putative neuroblast lineages in Aedes in more detail and compare them with the neuroblast lineages in Drosophila in order to determine if and to what extent clonal neural lineages are a general and conserved feature in the development of the fly brain.

Serotonin is known to play a very important role in the chemosensory system and in the feeding behavior of insects (Siju et al. 2008; Davis 1987; van Haeften and Schooneveld 1993; Lange et al. 1988; Nässel and Elekes 1984). The serotonergic system in the adult Aedes has been extensively studied and well documented by Siju et al. (2008). It consists of a set of six to eight pairs of serotonin positive neuronal somata in the subesophageal ganglion. Here, we characterize the development of these neural cells in the larval, $24 \mathrm{~h}$ APF pupal and adult stages of Aedes as a first step towards a more comprehensive analysis of the cellular and molecular mechanisms that underlie their development. Perhaps understanding how these specific neurons develop, as well as understanding development of the entire subesophageal ganglion, may help to increase our knowledge of host seeking and feeding behavior in these vector insects.

Acknowledgments We are very grateful to GM Technau, JB Skeath, CY Lee, J Pielage, and the Developmental Studies Hybridoma Bank (DSHB) for the antibodies. We also thank all the members of the Reichert lab for discussions and assistance. We are very thankful to the two unknown reviewers for their valuable comments on the previous version of the manuscript, their constructive criticism have greatly improved the manuscript. We are very grateful to Danica Jančariová and Mohamad Sater at Vector Control Centre, STPHI, Basel, Switzerland for insect rearing. This work was supported by the Indo-Swiss Joint Research Project (ISJRP).

\section{References}

Anton S, van Loon JJ, Meijerink J, Smid HM, Takken W, Rospars JP (2003) Central projections of olfactory receptor neurons from single antennal and palpal sensilla in mosquitoes. Arthropod Struct Dev 32:319-327

Arensburger P, Megy K, Waterhouse RM, Abrudan J, Amedeo P et al (2010) Sequencing of Culex quinquefasciatus establishes a platform for mosquito comparative genomics. Science 330:86-88

Bohbot J, Pitts RJ, Kwon HW, Rutzler M, Robertson HM, Zwiebel LJ (2007) Molecular characterization of the Aedes aegypti odorant receptor gene family. Insect Mol Biol 16:525-537

Bello B, Holbro N, Reichert H (2007) Polycomb group genes are required for neural stem cell survival in postembryonic neurogenesis of Drosophila. Development 134:1091-1099

Carey AF, Wang G, Su CY, Zwiebel LJ, Carlson JR (2010) Odorant reception in the malaria mosquito Anopheles gambiae. Nature 464:66-71

Chitnis AB, Kuwada JY (1990) Axogenesis in the brain of zebrafish embryos. J Neurosci 10:1892-1905

Dacks AM, Christensen TA, Hildebrand JG (2006) Phylogeny of a serotonin-immunoreactive neuron in the primary olfactory center of the insect brain. J Comp Neurol 498:727-746

Davis NT (1987) Neurosecretory neurons and their projections to the serotonin neurohemal system of the cockroach Periplaneta americana (L.), and identification of mandibular and maxillary motor neurons associated with this system. J Comp Neurol 259:604-621 
Eriksson BJ, Budd GE (2000) Onychophoran cephalic nerves and their bearing on our understanding of head segmentation and stem-group evolution of Arthropoda. Arthropod Struct Dev 29:197-209

Feachem RG, Phillips AA, Targett GA, Snow RW (2010) Call to action: priorities for malaria elimination. Lancet 376:1517-1521

Fox AN, Pitts RJ, Robertson HM, Carlson JR, Zwiebel LJ (2001) Candidate odorant receptors from the malaria vector mosquito Anopheles gambiae and evidence of down-regulation in response to blood feeding. Proc Natl Acad Sci USA 98:14693-14697

Gaunt MW, Miles MA (2002) An insect molecular clock dates the origin of the insects and accords with paleontological and biogeographic landmarks. Mol Biol Evol 19:748-761

Ghaninia M, Hansson BS, Ignell R (2007a) The antennal lobe of the African malaria mosquito, Anopheles gambiae - innervation and three-dimensional reconstruction. Arthropod Struct Dev 36:23-39

Ghaninia M, Ignell R, Hansson BS (2007b) Functional classification and central nervous projections of olfactory receptor neurons housed in antennal trichoid sensilla of female yellow fever mosquitoes, Aedes aegypti. Eur J Neurosci 26:1611-1623

Groh C, Rössler W (2008) Caste-specific postembryonic development of primary and secondary olfactory centers in the female honeybee brain. Arthropod Struct Dev 37:459-468

Halter DA, Urban J, Rickert C, Ner SS, Ito K, Travers AA, Technau GM (1995) The homeobox gene repo is required for the differentiation and maintenance of glia function in the embryonic nervous system of Drosophila melanogaster. Development 121:317-332

Hartenstein V, Spindler S, Pereanu W, Fung S (2008) The development of the Drosophila larval brain. Adv Exp Med Biol 628:131

Harzsch S, Hansson BS (2008) Brain architecture in the terrestrial hermit crab Coenobita clypeatus (Anomura, Coenobitidae), a crustacean with a good aerial sense of smell. BMC Neurosci 9:58

Hill CA, Fox AN, Pitts RJ, Kent LB, Tan PL, Chrystal MA, Cravchik A, Collins FH, Robertson HM, Zwiebel LJ (2002) G proteincoupled receptors in Anopheles gambiae. Science 298:176-178

Hofbauer A, Campos-Ortega JA (1990) Proliferation pattern and early differentiation of the optic lobes in Drosophila melanogaster. Roux's Arch Dev Biol 198:264-274

Ignell R, Dekker T, Ghaninia M, Hansson BS (2005) Neuronal architecture of the mosquito deutocerebrum. J Comp Neurol 493:207-240

Ignell R, Hansson BS (2005) Projection patterns of gustatory neurons in the suboesophageal ganglion and tritocerebrum of mosquitoes. J Comp Neurol 492:214-233

Ishii Y, Kubota K, Hara K (2005) Postembryonic development of the mushroom bodies in the ant, Camponotus japonicus. Zool Sci 22:743-753

Iwai Y, Usui T, Hirano S, Steward R, Takeichi M, Uemura T (1997) Axon patterning requires DN-cadherin, a novel neuronal adhesion receptor, in the Drosophila embryonic CNS. Neuron 19:7789

Klagges BR, Heimbeck G, Godenschwege TA, Hofbauer A, Pflugfelder GO, Reifegerste R, Reisch D, Schaupp M, Buchner S, Buchner E (1996) Invertebrate synapsins: a single gene codes for several isoforms in Drosophila. J Neurosci 16:3154-3165

Kwon HW, Lu T, Rutzler M, Zwiebel LJ (2006) Olfactory responses in a gustatory organ of the malaria vector mosquito Anopheles gambiae. Proc Natl Acad Sci USA 103:13526-13531

Lange AB, Orchard I, Lloyd RJ (1988) Immunohistochemical and electrochemical detection of serotonin in the nervous system of the blood-feeding bug Rhodnius prolixus. Arch Insect Biochem Physiol 8:187-201

Larsen C, Shy D, Spindler S, Fung S, Younossi-Hartenstein A, Hartenstein V (2009) Patterns of growth, axonal extension and axonal arborization of neuronal lineages in the developing Drosophila brain. Dev Biol 335:289-304

Lu T, Qiu YT, Wang G, Kwon JY, Rutzler M, Kwon HW, Pitts RJ, van Loon JJ, Takken W, Carlson JR, Zwiebel LJ (2007) Odor coding in the maxillary palp of the malaria vector mosquito Anopheles gambiae. Curr Biol 17:1533-1544

Martinez-Hernandez A, Bell KP, Norenberg MD (1977) Glutamine synthetase: glial localization in brain. Science 195:1356-1358

Mayer G, Whitington PM, Sunnucks P, Pfluger HJ (2010) A revision of brain composition in Onychophora (velvet worms) suggests that the tritocerebrum evolved in arthropods. BMC Evol Biol $10: 255-264$

Meinertzhagen IA, Hanson TE (1993) In: Bate M, Arias AM (eds) The development of the optic lobe. Cold Spring Harbor, New York, pp 1363-1491

Mellanby K (1958) The alarm reaction of mosquito larvae. Entomol Exp Appl 1:153-160

Melo AC, Rützler M, Pitts RJ, Zwiebel LJ (2004) Identification of a chemosensory receptor from the yellow fever mosquito, Aedes aegypti, that is highly conserved and expressed in olfactory and gustatory organs. Chem Senses 29:403-410

Merritt RW, Dadd RH, Walker ED (1992) Feeding behavior, natural food, and nutritional relationships of larval mosquitoes. Annu Rev Entomol 37:349-374

Mysore K, Shyamala BV, Rodrigues V (2010) Morphological and developmental analysis of peripheral antennal chemosensory sensilla and central olfactory glomeruli in worker castes of Camponotus compressus (Fabricius, 1787). Arthropod Struct Dev 39:310-321

Nässel DR, Elekes K (1984) Ultrastructural demonstration of serotonin immunoreactivity in the nervous system of an insect (Calliphora erythrocephala). Neurosci Lett 48:203-210

Oland LA, Tolbert LP (1996) Multiple factors shape development of olfactory glomeruli: insights from an insect model system. J Neurobiol 30:92-109

Olsson B, Klowden MJ (1998) Larval diet affects the alarm response of Aedes aegypti (L.) mosquitoes (Deiptera: Culicidae). J Insect Behav 11:593-596

Pereanu W, Younossi-Hartenstein A, Lovick J, Spindler S, Hartenstein V (2011) Lineage-based analysis of the development of the central complex of the Drosophila brain. J Comp Neurol 519:661-689

Preuss U, Landsberg G, Scheidtmann KH (2003) Novel mitosisspecific phosphorylation of histone $\mathrm{H} 3$ at $\mathrm{Thr} 11$ mediated by Dlk/ZIP kinase. Nucleic Acids Res 31:878-885

Rein K, Zockler M, Mader MT, Grubel C, Heisenberg M (2002) The Drosophila standard brain. Curr Biol 12:227-231

Rodrigues V, Hummel T (2008) Development of the Drosophila olfactory system. Adv Exp Med Biol 628:82-101

Siju KP, Hansson BS, Ignell R (2008) Immunocytochemical localization of serotonin in the central and peripheral chemosensory system of mosquitoes. Arthropod Struct Dev 37:248-259

Snow PM, Patel NH, Harrelson AL, Goodman CS (1987) Neural-specific carbohydrate moiety shared by many surface glycoproteins in Drosophila and grasshopper embryos. J Neurosci 7:4137-4144

Spindler SR, Hartenstein V (2010) The Drosophila neural lineages: a model system to study brain development and circuitry. Dev Genes Evol 220:1-10

Sprecher SG, Cardona A, Hartenstein V (2011) The Drosophila larval visual system: high-resolution analysis of a simple visual neuropil. Dev Biol. doi:10.1016/j.ydbio.2011.07.006

van der Hel WS, Notenboom RG, Bos IW, van Rijen PC, van Veelen CW, de Graan PN (2005) Reduced glutamine synthetase in hippocampal areas with neuron loss in temporal lobe epilepsy. Neurology 64:326-333

van Haeften T, Schooneveld H (1993) Diffuse serotoninergic neurohemal systems associated with cerebral and subesophageal nerves in the 
head of the Colorado potato beetle Leptinotarsa decemlineata. Cell Tissue Res 273:327-333

Wang G, Carey AF, Carlson JR, Zwiebel LJ (2010) Molecular basis of odor coding in the malaria vector mosquito Anopheles gambiae. Proc Natl Acad Sci USA 107:4418-4423

Ward MM, Jobling AI, Puthussery T, Foster LE, Fletcher EL (2004) Localization and expression of the glutamate transporter, excitatory amino acid transporter 4 , within astrocytes of the rat retina. Cell Tissue Res 315:305-310

Yeates DK, Wiegmann BM (1999) Congruence and controversy: toward a higher-level phylogeny of Diptera. Annu Rev Entomol 44:397-428
Zdobnov EM, von Mering C, Letunic I, Torrents D, Suyama M, Copley RR, Christophides GK, Thomasova D, Holt RA, Subramanian GM, Mueller HM, Dimopoulos G, Law JH, Wells MA, Birney E, Charlab R, Halpern AL, Kokoza E, Kraft CL, Lai Z, Lewis S, Louis C, Barillas-Mury C, Nusskern D, Rubin GM, Salzberg SL, Sutton GG, Topalis P, Wides R, Wincker P, Yandell M, Collins FH, Ribeiro J, Gelbart WM, Kafatos FC, Bork P (2002) Comparative genome and proteome analysis of Anopheles gambiae and Drosophila melanogaster. Science 298:149-159

Zipursky SL, Venkatesh TR, Teplow DB, Benzer S (1984) Neuronal development in the Drosophila retina: monoclonal antibodies as molecular probes. Cell 36:15-26 